\title{
An atomistic-based finite deformation membrane for single layer crystalline films
}

\author{
Marino Arroyo ${ }^{1}$, Ted Belytschko* \\ Department of Mechanical Engineering, Northwestern University, 2145 Sheridan Road, Evanston, \\ IL 60208-3111, USA
}

Received 18 August 2001; accepted 11 December 2001

\begin{abstract}
A general methodology to develop hyper-elastic membrane models applicable to crystalline films one-atom thick is presented. In this method, an extension of the Born rule based on the exponential map is proposed. The exponential map accounts for the fact that the lattice vectors of the crystal lie along the chords of the curved membrane, and consequently a tangent map like the standard Born rule is inadequate. In order to obtain practical methods, the exponential map is locally approximated. The effectiveness of our approach is demonstrated by numerical studies of carbon nanotubes. Deformed configurations as well as equilibrium energies of atomistic simulations are compared with those provided by the continuum membrane resulting from this method discretized by finite elements.
\end{abstract}

Keywords: A. Buckling; B. Constitutive behavior; B. Shells and membranes; C. Finite elements; D. Atomistic models

\section{Introduction}

The Born rule is a standard kinematic assumption for linking the deformation of an atomistic system to that of a continuum. It is the basis for developing continuum elastic potentials from the atomistic description of the system (Cousins, 1978; Ericksen, 1984; Zanzotto, 1996), without other phenomenological input, and has proven very effective in space-filling crystals. It states that the crystal vectors defined by two nuclei deform according to the local deformation gradient. The resulting hyper-elastic models describe

\footnotetext{
* Corresponding author. Tel.: +1-847-491-4029; fax: +1-847-491-4011.

E-mail addresses: m-arroyo@northwestern.edu (M. Arroyo), tedbelytschko@northwestern.edu (T. Belytschko).

${ }^{1}$ The support of the "la Caixa" Graduate Program is gratefully acknowledged.
} 
well the crystal behavior as long as the continuum deformation is nearly homogeneous in the scale of the crystal vectors. If this is not the case, mixed continuum-atomistic approaches have been proposed to deal with inhomogeneities, defects and non-local effects (Tadmor et al., 1996; Shenoy et al., 1999).

Unfortunately, the traditional approach based on the Born rule works for bulk materials but fails to extend directly to the case of crystalline films and ropes one-atom thick deforming in higher dimensional spaces, i.e. 3D in the case of films and 2D or $3 \mathrm{D}$ in the case of ropes. The reason for this can be roughly stated as follows: the deformation gradient on a surface gives the tangent behavior, whereas the deformation of the bonds depends on the behavior of the chords to the surface. This point is clarified later. It is the goal of this paper to extend the Born rule to this situation. The proposed extension is based on the differential geometry concept of the exponential map. An alternative approach resulting in a Cosserat membrane has been reported by Friesecke and James (2000) although their theory is aimed at films several atoms thick.

We will apply these methods to carbon nanotubes. However, they are applicable in other situations, such as boron-nitride nanotubes and other thin films. Since the discovery of these crystalline tubes in 1991, many studies have focused on their unique mechanical properties, through experiments (Yu et al., 2000; Chopra et al., 1995; Yu et al., 2001b), molecular dynamics (MD) and molecular mechanics (MM) simulations (Bernholc et al., 1998; Gad et al, 1998)
2001; Maiti, 2000). Although molecular
systems, they are not completely satisfacto
and the output often requires considerable
Continutum models have been used to exp

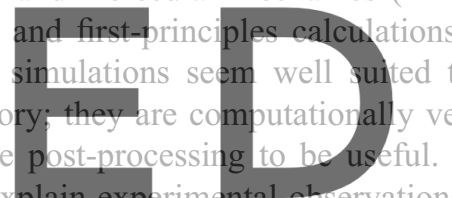

verse stability of nanotubes (Chopra et al., 1995; Yu et al., 2001a). These are extremely

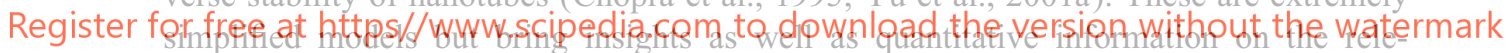

vant physical phenomena. The vibrational properties of carbon nanotubes have been investigated through linear elasticity (Sohlberg et al., 1998). The elastic properties of carbon nanotubes as a continuum, neglecting all curvature effects have been studied by $\mathrm{Lu}$ (1997). In Zhong-can et al. (1997), the nanotube is considered to be an inextensible membrane and an expression for the elastic energy in terms of the curvature obtained for a family of simple deformations. The theory of elastic shells is used to study the buckling patterns found in MD simulations of compressed carbon nanotubes by Yakobson et al. (1996). A linearized bifurcation analysis of the continuum shell predicts critical strains for the nanotube that agree to some extent with those observed in MD simulations. Ru (2001) describes a similar linearized buckling analysis of double-walled nanotubes.

The continuum models for nanotubes proposed so far are phenomenological and simplified to very particular situations. Furthermore, they are restricted to small strains. However, experiments (Chopra et al., 1995) and first-principles calculations (Maiti, 2000) show that carbon nanotubes undergo very large deformations, with highly nonlinear behavior and still remain elastic, with stable bonds and intact bond topology. Experiments of nanotubes severely deforimed with an AFM tip (Falvo et al., 1997) show the resilience and flexibility of these molecules; they recover elastically from extremely bent and buckled configurations. Atomistic simulations of nanotubes in tension 
show that large strains are reached before the Stone-Wales transformations occur (Bernholc et al., 1998; Belytschko et al., 2001). Thus, a Born-like (and therefore based on the nano-scale physics) finite deformation elastic model should be applicable to a large range of phenomena. It is our aim to develop such a model for curved crystalline films.

The paper is organized as follows. In Section 2, we first give the motivation for the extended Born rule. Section 3 presents the kinematics of the membrane and differential geometry preliminaries. Section 4 describes the proposed Exponential Born rule, and its practical implementation by suitable approximations for the exponential map is described in Section 5. Section 6 deals with the formulation of the continuum membrane model. The continuum strain energy density is constructed for simple Bravais lattices and Bravais multi-lattices. The latter requires the treatment of additional internal variables. The continuum version of the non-bonded interactions is also provided, as well as the variational statement of the continuum problem. Section 7 gives some examples of calculations made with our model discretized with finite elements. Finally, some conclusions and observations on this method are given in Section 8.

\section{Standard Born rule for space-filling crystals}
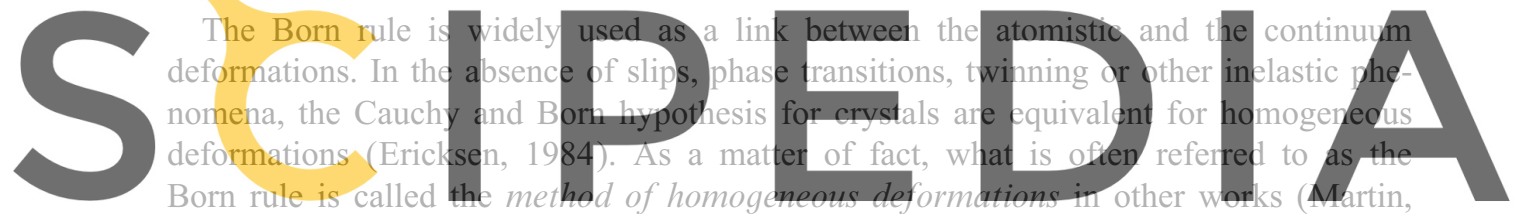

1975; Cousins, 1978). Cauchy linked atomic models to continua by assuming that the

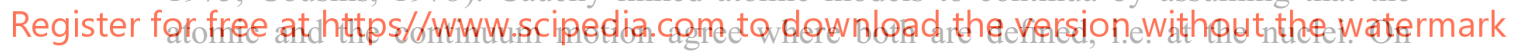

the other hand, the Born hypothesis consists of assuming the deformation of the lattice vectors conforms to that of material line elements in a homogeneous deformation.

\subsection{The Born rule}

The Born rule is usually expressed in terms of the deformation gradient $\mathbf{F}$. In classical continuum mechanics, the deformation gradient is said to map "infinitesimal" material vectors, $\mathrm{d} \mathbf{x}=\mathbf{F} \mathrm{d} \mathbf{X}$ from the undeformed body $\Omega_{0}$ into the deformed one $\Omega$ (Malvern, 1969). The Born rule views the lattice vectors as infinitesimal material vectors that transform according to

$$
\mathbf{a}=\mathbf{F A}
$$

where $\mathbf{A}$ denotes an undeformed lattice vector and a the same vector in the deformed crystal. From a physical standpoint, the lattice vectors are physical entities with finite length, and therefore the rule expressed by Eq. (1) entails an approximation. It is applicable as long as the continuum deformation is nearly homogeneous in the scale of the lattice vectors. 3

The geometry of the lattice vectors, that is their length and the angles they form with other lattice vectors in the deformed crystal, can be extracted from the continuum 
deformation through the Green deformation tensor $\mathbf{C}=\mathbf{F}^{\mathrm{T}} \mathbf{F}$ using standard continuum mechanics relations:

$$
\|\mathbf{a}\|=\sqrt{\mathbf{A} \cdot \mathbf{C A}} \text { and } \cos \theta=\frac{\mathbf{A} \cdot \mathbf{C B}}{\|\mathbf{a}\|\|\mathbf{b}\|},
$$

where $\mathbf{B}$ and $\mathbf{b}$ represent another undeformed and deformed lattice vectors and $\theta$ is the angle $\mathbf{a}$ and $\mathbf{b}$ form in the deformed crystal. Once the geometry of the deformed lattice vectors is linked to the continuum deformation, a constitutive model based on the atomistic description can be constructed by identifying the continuum strain energy density with the potential energy of the atomic system for a representative cell divided by its volume.

\subsection{Why films are more difficult}

Consider the case in which we have a single-atom thick crystalline film (such as a graphene sheet) deforming arbitrarily in 3D. It is natural in this case to treat the continuum solid as a surface, a membrane without thickness. We postulate that the atoms lie on the surface (Cauchy's hypothesis), and the lattice vectors are chords of the surface. We would like to use the Born rule in order to express the geometry of the deformed latice vedtors in terms of a variable characterizing the deformation of the surface, such as the Green deformation tensor.

If we now try to use directly the Born encountered is that case of surfaces.
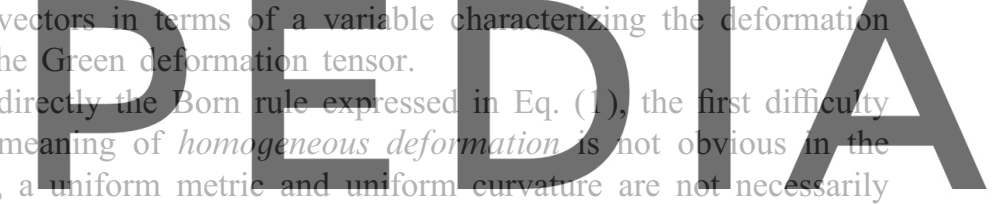

compatible, i.e. there may not exist a surface with such uniform properties (do Carmo,

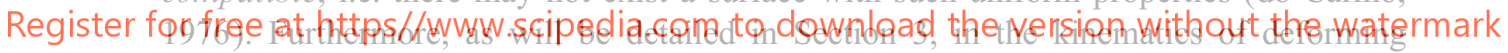
surfaces, the deformation gradient $\mathbb{F}$ maps the tangent spaces of the surfaces, $T_{\mathbf{X}} \Omega_{0}$ and $T_{\mathbf{x}} \Omega$ (see Fig. 1 for an illustration). For surfaces, the notation $T_{p} S$ represents the tangent plane to the smooth surface $S$ at the point $p \in S$. The tangent space is

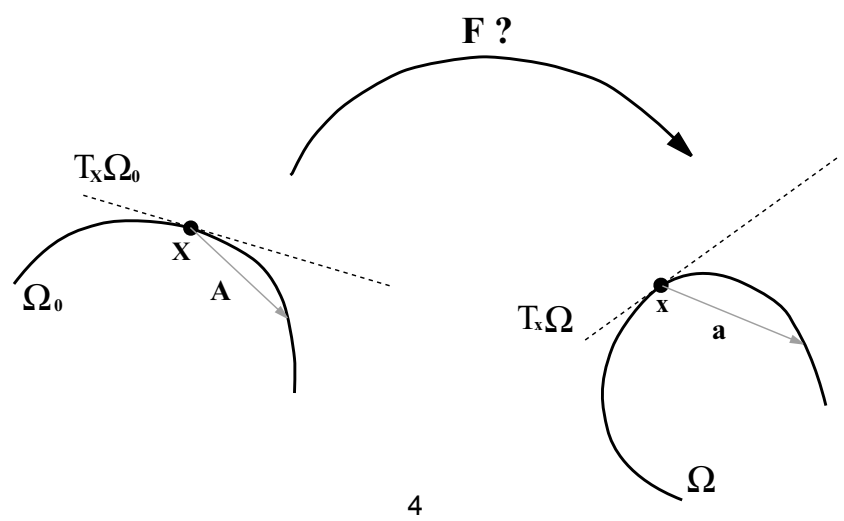

Fig. 1. Illustration of the difficulties encountered when trying to apply the standard Born rule $\mathbf{a}=\mathbf{F A}$ to surfaces. 
then the vector space $\mathbb{R}^{2}$ tangent to the surface and "centered" at $p$. Here, unlike for space-filling materials, the manifold and its tangent space cannot be identified; there is an essential distinction between the manifold, which is curved, and the tangent space, which is flat. As noted, the lattice vectors should be viewed as chords of the surface, not as elements of the tangent to the surfaces. From Fig. 1, it is clear then that the deformation gradient cannot be used to transform the undeformed lattice vector $\mathbf{A}$ into the deformed lattice vector $\mathbf{a}$.

To illustrate these issues, consider the following situation. Suppose that the undeformed crystal is planar, and is rolled into a cylinder without stretch. If the standard Born rule is used, the deformed lattice vectors emanating from the same point remain coplanar, i.e. they lie in the tangent space to the cylinder at that point. In this tangent space, energetically relevant geometric quantities such as the length of the lattice vectors and the angles they make remain unchanged (the deformation described is an isometry). Therefore, the energy will remain unchanged upon rolling. However, the real lattice vectors do not remain coplanar and their lengths change. Therefore, we can expect that the energy of the atomic system will change in this deformation. Thus, a continuum model describing only the behavior of the tangent space is blind to the fact that the plane sheet is being rolled, and assigns zero energy to the deformation.
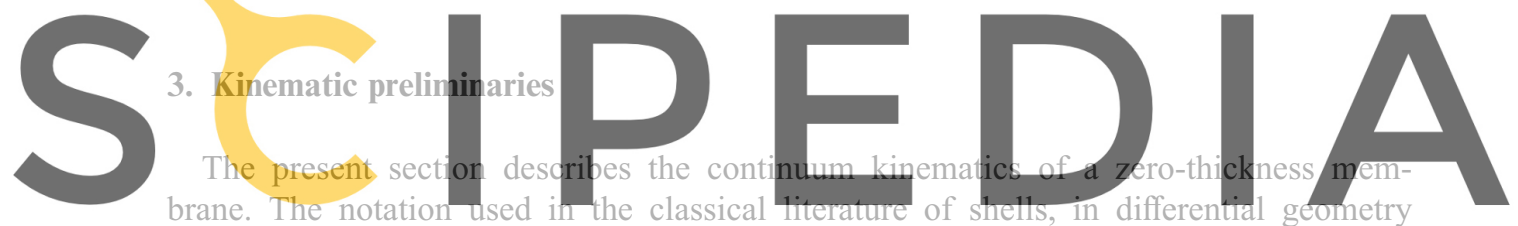
and in continuum mechanics is very different despite sharing many. underlying conRegister for free at httpskbww scipedia com to down had the versjon withoyt the watermark section are Marsden and Hughes (1983) for a mathematical treatment of continuum mechanics, and do Carmo (1976) for the differential geometry of curves and surfaces.

\subsection{The deformation map}

If the undeformed body is an arbitrary smooth surface, we can formulate the kinematics of the membrane as follows. Consider the undeformed body $\Omega_{0}$ to be defined through a differentiable parametrization of a referential body $\bar{\Omega}$, an open set in $\mathbb{R}^{2}$ (which can be called the parametric space). This parametrization is called the undeformed configuration $\varphi_{0}$ :

$$
\begin{aligned}
\varphi_{0}: \bar{\Omega} \subset \mathbb{R}^{2} & \rightarrow \mathbb{R}^{3} \\
\boldsymbol{\xi} & \mapsto \mathbf{X}=\varphi_{0}(\boldsymbol{\xi}),
\end{aligned}
$$

where $\xi$ represents any point in $\bar{\Omega}$ and $\mathbf{X}$ its image in the deformed body. The undeformed body $\Omega_{0}=\varphi_{0}(\bar{\Omega})$ is a 2-differential manifold (a smooth surface) embedded in the ambient space $\mathbb{R}^{3}$.

Similarly, the deformed body $\Omega$ is another 2-differential manifold in $\mathbb{R}^{3}$, and it is parametrized on the same referential body $\bar{\Omega}$ by the current or deformed 


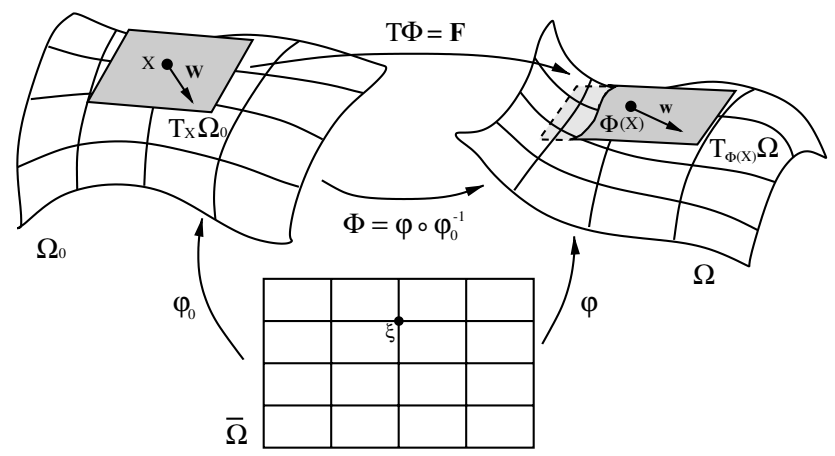

Fig. 2. Deformation man and its tangent map for surfaces in $3 \mathrm{D}$

\section{configuration}
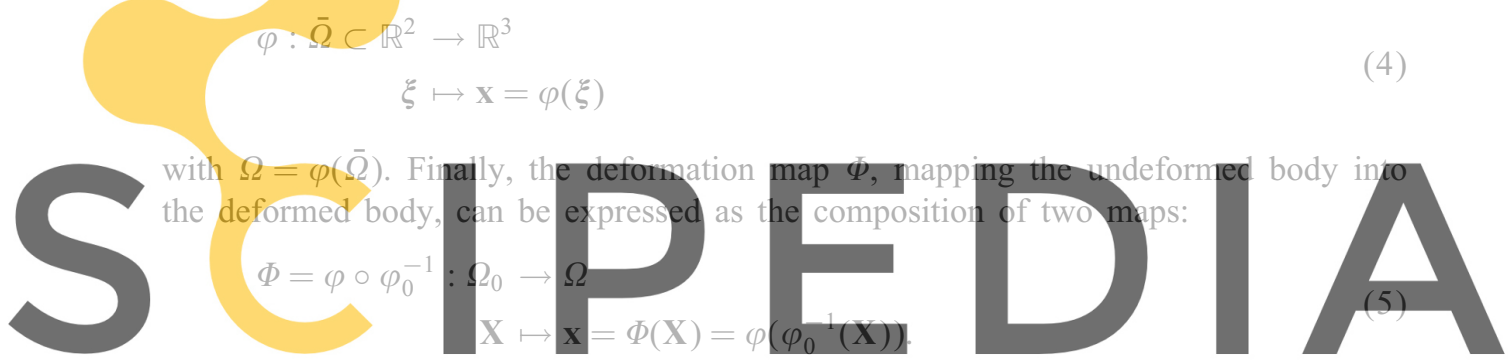

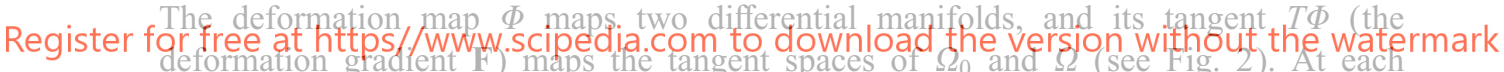
deformation gtadient $1 F)$ maps the tangent spaces of $\Omega_{0}$ and $\Omega($ see Fig. 2). At each

point $\mathbf{X}$ of $\Omega_{0}, T_{\mathbf{X}} \Phi=\mathbb{F}(\mathbf{X})$ is a linear transformation taking elements of $T_{\mathbf{X}} \Omega_{0}$ (the vector space whose elements lie in the tangent plane to the surface $\Omega_{0}$ at $\mathbf{X}$ and emanate from this point) into $T_{\Phi(\mathbf{X})} \Omega$ (analogously, the vector space tangent to the deformed surface at $\Phi(\mathbf{X})$ "centered" at this same point). This geometric structure is hidden in the case of bulk materials described with Euclidean coordinates. See the references given at the beginning of this section for more details on these concepts.

For the sake of simplicity, the parametric space or referential body is described here by Euclidean coordinates. Therefore, in the referential body the distinction between one-forms and vectors, or cova- and contra- components vanishes (the metric of the referential body is $\left.\bar{G}_{I J}=\bar{G}^{I J}=\delta_{I J}\right)$. On the other hand, we will denote the point and its position vector in Euclidean components with the same symbol

$$
\boldsymbol{\xi}=\xi^{1} \mathbf{I}_{1}+\xi^{2} \mathbf{I}_{2}
$$

Similarly, $\varphi_{0}$ and $\varphi$ denote both the point mappings and their Euclidean components, and we can write for instance,

$$
\varphi=\varphi^{1} \mathbf{i}_{1}+\varphi^{2} \mathbf{i}_{2}+\varphi^{3} \mathbf{i}_{3},
$$

where $\left\{\mathbf{i}_{i}\right\}$ represents the Euclidean basis of $\mathbb{R}^{3}$. 


\subsection{The metric tensor and the tangents of the configurations}

Given the Euclidean coordinate system for $\bar{\Omega}$ and its basis vectors $\left\{\mathbf{I}_{I}\right\}$, the natural or convected basis vectors (tangent vectors to the surfaces $\Omega_{0}$ and $\Omega$ respectively) are defined as

$$
\mathbf{G}_{I}=\frac{\partial \varphi_{0}^{i}}{\partial \xi^{I}} \mathbf{i}_{i} \quad \text { and } \quad \mathbf{g}_{I}=\frac{\partial \varphi^{i}}{\partial \xi^{I}} \mathbf{i}_{i}
$$

Then, at each point $\mathbf{X}$ and $\mathbf{x},\left\{\mathbf{G}_{1}, \mathbf{G}_{2}\right\}$ is a basis of $T_{\mathbf{X}} \Omega_{0}$ and $\left\{\mathbf{g}_{1}, \mathbf{g}_{2}\right\}$ of $T_{\mathbf{x}} \Omega$. The corresponding dual bases of the cotangent spaces of $\Omega_{0}$ and $\Omega$ are also defined. The cotangent space $T_{\mathbf{x}}^{*} \Omega$ is the space of one-forms on $T_{\mathbf{x}} \Omega$, i.e. the linear mappings from the tangent into $\mathbb{R}$. The dual basis is defined by the relations $\mathbf{g}^{a}\left(\mathbf{g}_{b}\right)=\delta_{b}^{a}$ (analogous relations hold for the undeformed body).

The metric tensor of the surfaces can then be defined by its covariant components (the components in the bases $\left\{\mathbf{G}^{A} \otimes \mathbf{G}^{B}\right\}$ and $\left\{\mathbf{g}^{a} \otimes \mathbf{g}^{b}\right\}$, respectively):

$$
G_{A B}=\left\langle\mathbf{G}_{A} \mid \mathbf{G}_{B}\right\rangle \text { and } g_{a b}=\left\langle\mathbf{g}_{a} \mid \mathbf{g}_{b}\right\rangle \text {, }
$$

where $\langle\cdot \mid \cdot\rangle$ is the scalar product in $T \Omega_{0}$ and in $T \Omega$ induced by the Euclidean scalar

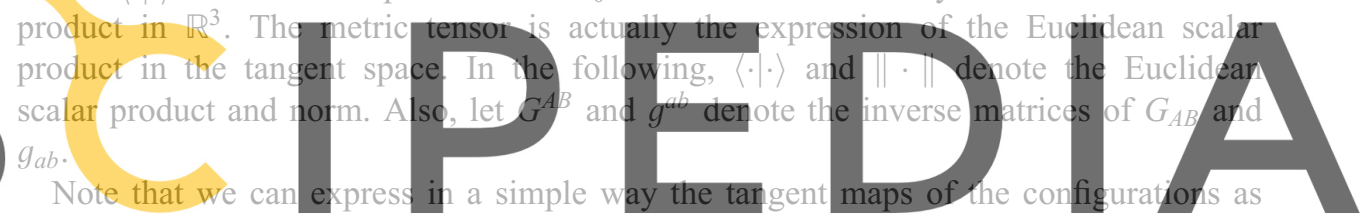

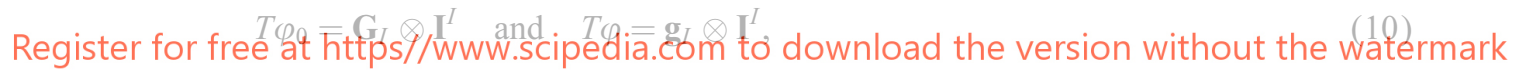

where $\left\{\mathbb{I}^{I}\right\}$ is the dual basis of $\left\{\mathbb{I}_{I}\right\}$.

The metric tensor can be used to measure distances, angles and areas in $\Omega_{0}$ and $\Omega$. In particular the element of area of $\Omega$ is $\mathrm{d} \Omega=g \mathrm{~d} \xi^{1} \mathrm{~d} \xi^{2}$ where $g$ is defined by

$$
g=\sqrt{\operatorname{det}\left(g_{a b}\right)}=\left\|\mathbf{g}_{1} \times \mathbf{g}_{2}\right\| .
$$

We also have $\mathrm{d} \Omega_{0}=G \mathrm{~d} \xi^{1} \mathrm{~d} \xi^{2}$ with

$$
G=\sqrt{\operatorname{det}\left(G_{A B}\right)}=\left\|\mathbf{G}_{1} \times \mathbf{G}_{2}\right\| .
$$

\subsection{The deformation gradient}

Recalling the definition of the deformation map $\Phi=\varphi \circ \varphi_{0}^{-1}$ and Eq. (10), we can write its tangent map $T \Phi=T \varphi \circ T \varphi_{0}^{-1}$, or deformation gradient as

$$
\begin{aligned}
\mathbf{F}=\mathbf{g}_{A} \otimes \mathbf{G}^{A}: T \Omega_{0} & \rightarrow T \Omega \\
\mathbf{W}=W^{I} \mathbf{G}_{I} & \mapsto \mathbf{w}=\mathbf{F W}=W_{7}^{i} \mathbf{g}_{i} .
\end{aligned}
$$

Therefore, the matrix representation of $\mathbf{F}$ in the convected bases of $T_{\mathbf{X}} \Omega_{0}$ and $T_{\mathbf{X}} \Omega$ is the $2 \times 2$ identity matrix, with the information about the deformation contained in the 
basis vectors. Recalling that the components of $\mathbf{F}$ in the basis $\left\{\mathbf{g}_{a} \otimes \mathbf{G}^{A}\right\}$ are $F^{a}{ }_{A}=$ $\delta^{a}{ }_{A}$, the components of the Green deformation tensor $\mathbf{C}=\mathbf{F}^{\mathrm{T}} \mathbf{F}$ in the convected basis $\left\{\mathbf{G}_{A} \otimes \mathbf{G}^{B}\right\}$ are (see Marsden and Hughes, 1983)

$$
C_{B}^{A}=G^{A C} F^{b}{ }_{C} g_{a b} F^{a}{ }_{B}=G^{A b} g_{b B} .
$$

The Green deformation tensor with lowered indices $\mathbf{C}^{b}$ is the pull-back of the metric tensor of $\Omega, \mathbf{C}^{b}=\Phi^{*} \mathbf{g}$, and its components are simply $C_{A B}=g_{A B}$ for our choice of coordinates. It is a symmetric positive-definite tensor, and therefore defines a scalar product in $T \Omega_{0}$. We denote this scalar product by

$$
\langle\mathbf{A} \mid \mathbf{B}\rangle_{\mathbf{C}}=\mathbf{A} \mathbf{C}^{b} \mathbf{B}=\mathbf{A} \cdot \mathbf{C B},
$$

for all $\mathbf{A}, \mathbf{B} \in T \Omega_{0}$. The norm induced by this scalar product is denoted by $\|\mathbf{A}\|_{C}=$ $\sqrt{\langle\mathbf{A} \mid \mathbf{A}\rangle_{\mathrm{C}}}$. Thus, the tensor $\mathbf{C}$ locally characterizes the tangent deformation: it can be used to measure length, angle and area changes due to the deformation in terms of undeformed body entities. In particular, the element of area of $\Omega$ can be written in terms of the element of area of $\Omega_{0}$ as $\mathrm{d} \Omega=J \mathrm{~d} \Omega_{0}$ where the Jacobian determinant is
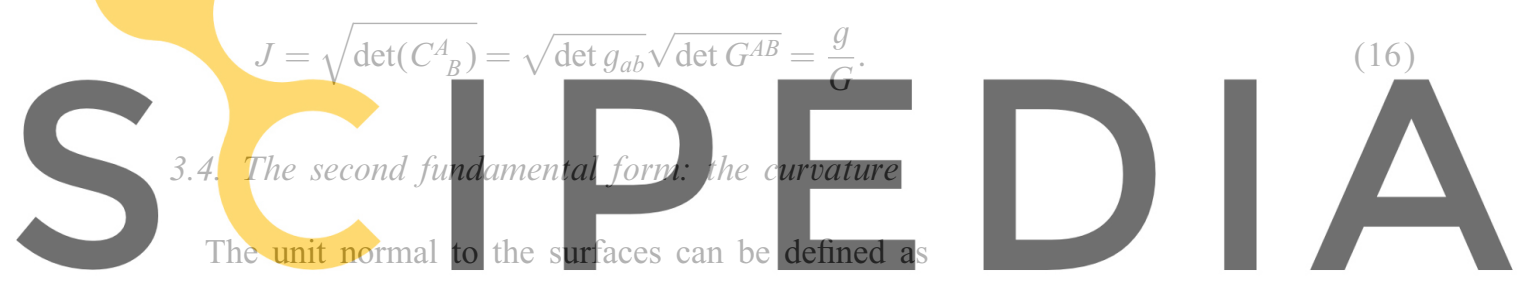

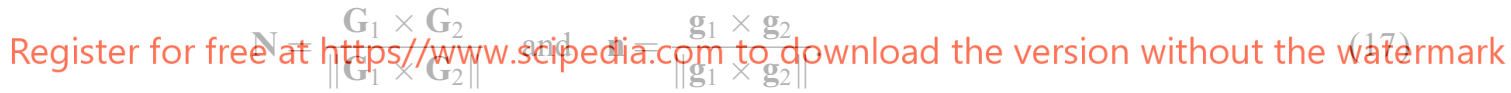

The second fundamental form of the undeformed body $\mathbf{K}$ can be expressed in the basis $\left\{\mathbf{G}^{A} \otimes \mathbf{G}^{B}\right\}$ in terms of its components:

$$
K_{A B}=\left\langle\mathbf{N} \mid \mathbf{G}_{A, B}\right\rangle,
$$

where $\mathbf{G}_{A, B}$ denotes the derivative of the tangent vector $\mathbf{G}_{A}$ with respect to $\xi^{B}$. Similarly, for the deformed body, the components of $\mathbf{k}$ in the basis $\left\{\mathbf{g}^{a} \otimes \mathbf{g}^{b}\right\}$ are

$$
k_{a b}=\left\langle\mathbf{n} \mid \mathbf{g}_{a, b}\right\rangle .
$$

Let us define now the normal curvature in the deformed body. The normal curvature $k_{n}$ at a point $\mathbf{x}$ of the surface $\Omega$ and in the direction of the unit vector $\mathbf{v}=v^{a} \mathbf{g}_{a} \in T_{\mathbf{x}} \Omega$, is the minimum curvature of all the curves of $\Omega$ passing through $\mathbf{x}$ tangent to $\mathbf{v}$. It can be obtained as

$$
k_{n}(\mathbf{x})=k_{a b} v^{a} v^{b} \quad \text { with } \quad g_{a b} v^{a} v^{b}=1 .
$$

Consider now the pull-back by $\Phi$ of the Eurvature tensor of $\Omega$ :

$$
\mathscr{K}=\Phi^{*} \mathbf{k},
$$


or in components:

$$
\mathscr{K}_{A B}=k_{a b} F_{A}^{a} F^{b}{ }_{B} .
$$

The normal curvature of the deformed body in a given direction expressed in the undeformed configuration $\mathbf{V} \in T_{\mathbf{X}} \Omega_{0}$ can be written as follows:

$$
k_{n}=\frac{\mathbf{V} \mathscr{K} \mathbf{V}}{\mathbf{V C}^{b} \mathbf{V}} .
$$

The principal curvatures $k_{I, I I}$ and the principal directions $\mathbf{V}_{I, I I}$ of the curvature tensor are the eigenvalues and the eigenvectors of the generalized eigenvalue problem:

$$
\mathscr{K} \mathbf{V}_{I, I I}=k_{I, I I} \mathbf{C}^{b} \mathbf{V}_{I, I I} \text {. }
$$

Note that the principal directions are expressed in the undeformed configuration. The principal curvatures are the maximum and the minimum normal curvatures, and the principal directions $\mathbf{V}_{I, I I}$ are orthogonal with respect to $\mathbf{C}^{b}$. Finally, the mean curvature $H$ and the Gaussian curvature $K$ are defined as

$$
H=\frac{k_{I}+k_{I I}}{2} \text { and } K=k_{I} k_{I I}
$$

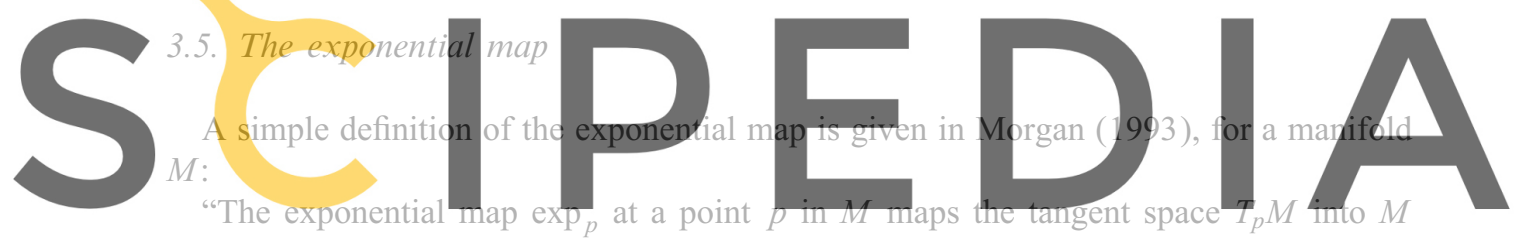

by sending a vector $\mathbf{v}$ in $T_{n} M$ to the point in $M$ a distance $|\mathbf{v}|$ along the geodesic from Register for freq at.https/./Www.scipedia.com to download the version without the watermark

The exponential map is a diffeomorphism - invertible and differentiable - in a neighborhood of each regular point $p$ of the manifold, that naturally maps the tangent of a manifold into the manifold itself in an intrinsic manner. Therefore, the exponential map can be used to locally parametrize the surface, defining the polar geodesic coordinates. It can be defined because of the existence and uniqueness of geodesics at any point given a direction in the tangent space. The exponential map is defined here in geometric terms, and its evaluation requires knowledge of the geodesics. In general, obtaining the geodesics in a particular coordinate system involves solving the geodesic differential equations. These equations are a system of non-linear ordinary differential equations whose unknowns are the parametric coordinates of the geodesic, and whose coefficients are the Christoffel symbols of the surface. Finding the geodesics, and thus the exponential map, is much simpler in some particular cases, as will be shown for the cylinder. More details about the exponential map in the case of surfaces can be found in do Carmo (1976).

It is worth commenting on another important application of the exponential map in computational mechanics: the transformation of infinitesimal rotations (represented by skew-symmetric matrices or the corresponding spin vector) into finite rotations (represented by orthogonal proper matrices) (Simo and Hughes, 1998). The underlying idea is very similar to the one used here. Indeed, the infinitesimal rotations are the 
tangent to the finite rotations. Further geometrical insight is gained by understanding the correspondence between the finite rotations and the unit sphere (Simo and Fox, 1989).

\section{Extension of the Born rule}

In order to construct a continuum mechanics theory from an atomistic model, we need to distribute the deformation energy of the discrete atomic system into the continuum. For this purpose, following the path of crystal elasticity, we need an analog of the standard Born rule that relates the deformation of the lattice vectors to that of the continuum membrane.

In Section 2.2, the breakdown of the standard Born rule for curved continua was sketched. We saw that the kinematic rule $\mathbf{a}=\mathbf{F A}$ presented formal inconsistencies. In this section, these are overcome by introducing a new kinematic rule that exploits the exponential map. In this section, the proposed rule is kept at an abstract level, and should be seen as a framework for practical models. Reference to Fig. 3 is particularly useful to visualize the ideas presented below.

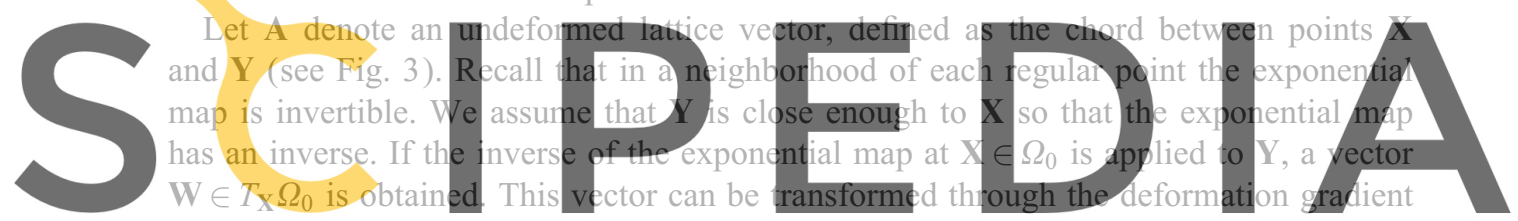

$\mathbb{F}$ into a vector $\mathrm{w} \in T_{\mathrm{x}} \Omega$, which in turn can be sent back to a point $\mathbb{Z} \in \Omega$ through the

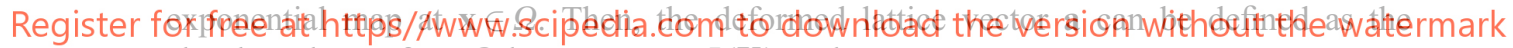
chord to the surface $\Omega$ between $\mathbf{x}=\Phi(\mathbf{X})$ and $\mathbb{Z}$.

In order to keep the notation simple and highlight the fundamental ideas, the following notation is used. The exponential map at $\mathbf{X} \in \Omega_{0}$ maps tangent vectors $\mathbf{W} \in T_{\mathbf{X}} \Omega_{0}$ into points in the surface $\mathbf{Y}=\exp _{\mathbf{X}}(\mathbf{W})$. Since, given $\mathbf{X}$, the chord $\mathbf{A}$ and the point $\mathbf{Y}$ can be identified, we will simply write $\mathbf{A}=\exp _{\mathbf{X}}(\mathbf{W})$. The same notation applies in the deformed body.

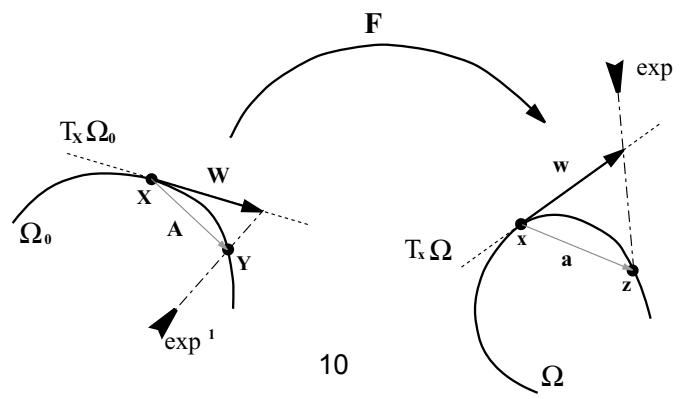

Fig. 3. Illustration of the proposed extension of the Born rule. 
Therefore, the proposed kinematic rule can be summarized as:

$$
\begin{aligned}
& \exp _{\Phi(\mathbf{X})} \circ \mathbf{F}(\mathbf{X}) \circ \exp _{\mathbf{X}}^{-1}: \Omega_{0} \rightarrow T_{\mathbf{X}} \Omega_{0} \quad \rightarrow \ldots \\
& \mathbf{A} \mapsto \mathbf{W}=\exp _{\mathbf{X}}^{-1}(\mathbf{A}) \mapsto \ldots \\
& \ldots T_{\Phi(\mathbf{X})} \Omega \quad \rightarrow \Omega \\
& \ldots \mathbf{w}=\mathbf{F W} \quad \mapsto \mathbf{a}=\exp _{\Phi(\mathbf{X})}(\mathbf{w}) .
\end{aligned}
$$

The above map overcomes the formal inconsistencies of the standard Born rule pointed out in Section 2.2 by exploiting the natural way to map the tangent space and the surface provided by the exponential map. This extension to the Born rule will be called Exponential Born rule in the remainder of the paper, and is symbolically denoted as

$$
\mathbf{a}=\mathscr{F}_{\Phi}(\mathbf{A}):=\exp _{\Phi(\mathbf{X})} \circ \mathbf{F}(\mathbf{X}) \circ \exp _{\mathbf{X}}^{-1}(\mathbf{A}) .
$$

This map transforms an undeformed lattice vector into a deformed one based on the deformation of the surface. In our model, the deformation gradient expresses changes in intrinsic length between the atoms (length inside the surface, defined as the length of the shortest curve between them on the surface), but not necessarily the actual Euclidean distance between the atoms, i.e. the length of the lattice vector.

Note that strictly speaking the map $\exp _{\Phi(\mathbf{X})} \circ \mathbf{F}(\mathbf{X}) \circ \exp _{\mathbf{X}}^{-1}$ is intrinsic, i.e. can be performed "from inside" the surface, without recourse to the ambient Euclidean space. However, the total map is necessarily extrinsic, because the energy is ultimately expressed in terms of lattice vectors in $\mathbb{R}^{3}$. The extrinsic part of the map stems from the definition of lattice vectors as chords of the surface.

Although this model provides a theoretical and formal way to overcome the previously mentioned difficulties of the standard Born rule, its practical application is not straight-forward. To determine the geodesics, and thus the exponential map, the geodesic differential equations must be integrated. The exact implementation of the proposed model results in a computationally very complex non-local model. Indeed, the deformed lattice vectors would depend not only on the deformation of the surface at a particular point, but in a neighborhood. However, approximations to the exponential map can be used, rendering the model possibly local and computationally feasible. One can consider the kinematic assumption $\mathbf{a}=\mathscr{F}_{\Phi}(\mathbf{A})$ as a general framework for a family of extensions to the Born rule in different situations. In Section 6, these abstract ideas are practically realized in a model applicable to carbon nanotubes.

Remark 1. If this model is applied to space-filling bodies, it results exactly in the standard Born rule. Indeed, the geodesics in a subset of the Euclidean space $\mathbb{R}^{n}$ are straight lines and the exponential map is, loosely speaking, an identity map.

Remark 2. It can be said that this is a higher order model, because it involves not only the metric but also its derivatives (the Christoffel symbols depend on the metric and its first derivatives). We prefer to think of it in the geometric terms presented. The present approach defined in geometric terms autonatically leads to hyper-elastic potentials that satisfy frame-indifference. A "higher order" ad hoc model will have difficulties in this respect. 
Remark 3. Note that in general $\mathbf{z} \neq \Phi(\mathbf{Y})$ (see Fig. 3). Actually, the same happens with the standard Born rule, unless the deformation is homogeneous. Similarly, in the present theory there are some special cases where (26) it is kinematically exact in this sense.

\section{Implementation of the exponential Born rule}

This sections addresses the implementation of the Exponential Born rule. For the sake of simplicity, we assume the undeformed body $\Omega_{0}$ is a planar crystalline sheet described with Euclidean coordinates. Therefore, the referential and the undeformed bodies coincide. In particular, we have $G_{A B}=\delta_{A B}, G=1$ and $\mathbf{K}=\mathbf{0}$. The choice of a planar undeformed body is natural for our application to carbon nanotubes, since usually the inter-atomic potentials available for graphene sheets are in equilibrium in the planar configuration.

In general the evaluation of the exponential map, and therefore the application of the extended rule, requires knowledge of the geodesic curves. In a given coordinate system, these are obtained by integration of a system of two non-linear ODE's. The coefficients of these equations are the Christoffel symbols. In general, a closed-form solution of these equations is not available, but one can think of approximations based on these equations. For instance, they could be solved numerically, or simplified so that a closed-form solution is available. These approaches have obvious drawbacks. The first one is the computational cost and complexity of the resulting method. Secondly, it would be difficult to guarantee frame-indifference.

In a paper dealing only with the transverse deformation of nanotubes (Arroyo and Belytschko, 2001), a geometric approach was adopted. In this simple kinematic setting, the surface was locally assumed to be a cylinder with the curvature and the stretch of the actual surface. Then, since the exponential map for the cylinder can be obtained in closed form, the deformation of the lattice vectors was written in terms of the local deformation of the kinematically constrained membrane.

We now consider an arbitrary deformation of the crystalline film. Rather than trying to build at each point of the membrane a local representation based on its local deformation, the exponential map is approximated by decoupling the principal directions. The procedure separately considers each principal direction $\mathbf{V}_{I}$ and $\mathbf{V}_{I I}$ of the curvature tensor, and two corrections for the tangent deformed lattice vector $\mathbf{w}$ are obtained from the exponential map for fictitious cylinders of radius $1 / k_{I}$ and $1 / k_{I I}$. Finally, the corrections in each direction are added to $\mathbf{w}$ to obtain $\mathbf{a}$. This approach is simple and leads to accurate predictions according to the numerical experiments described later.

\subsection{Basic setup}

For the planar undeformed crystal, the Exponential Born rule simplifies to

$$
\mathbf{a}=\exp _{\Phi(\mathbf{X})} \circ \mathbf{F}(\mathbf{X}) \mathbf{A}
$$




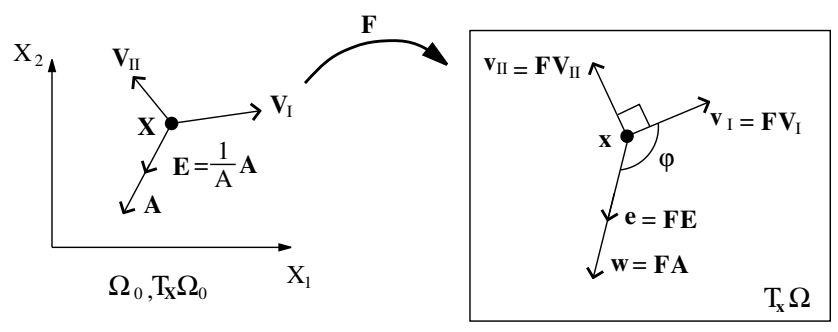

Fig. 4. Principal directions of the curvature tensor in the undeformed and the deformed bodies, and a lattice vector $\mathbf{A}$.

where we are identifying $\Omega_{0}$ and $T_{\mathbf{X}} \Omega_{0}$. The first part of this map can be readily performed and is equivalent to the standard Born rule:

$$
\mathbf{w}=\mathbf{F A} \text {. }
$$

We call this vector the tangent deformed lattice vector, and it can be thought as the push-forward of $\mathbf{A}$.

Given a deformation map for the originally planar membrane, the local deformation can be characterized by the Green deformation tensor $\mathbf{C}$ and the curvature tensor expressed in $\Omega_{0}, \mathscr{K}$. The eigenvalue problem (24) defines the principal directions expressed in the undeformed configuration and principal curvatures of the curvature tensor. Fig. 4 illustrates that only the push-forward of $\mathbf{V}_{I}$ and $\mathbf{V}_{I I}$ by $\Phi$, that is $\mathbf{v}_{I}$ and $\mathbf{v}_{I I}$, are orthogonal in the Euclidean sense. This figure also shows a generic undeformed lattice vector $\mathbf{A}$; a unit vector in the direction of $\mathbf{A}$ is also defined by

$$
\mathbf{E}=\frac{1}{A} \mathbf{A}
$$

where $A$ denotes the length of $\mathbf{A}$. Note that $\mathbf{v}_{I, I I}$ and $\mathbf{w}$ are vectors of the tangent to the membrane $T_{\Phi(\mathbf{X})} \Omega$.

Consider an auxiliary Euclidean coordinate system of $\mathbb{R}^{3},\left\{x_{I}, x_{I I}, x_{I I I}\right\}$ centered at $\mathbf{X}=\Phi(\mathbf{X})$ and whose axes are parallel to $\mathbf{v}_{I}, \mathbf{v}_{I I}$, and $\mathbf{v}_{I} \times \mathbf{v}_{I I}$. The associated orthonormal basis is $\mathscr{B}_{\mathbb{R}^{3}}=\left\{\mathbf{i}_{I}, \mathbf{i}_{I I}, \mathbf{i}_{I I I}\right\}$. Consider also the restriction of this coordinate system to $T_{\mathbf{x}} \Omega,\left\{x_{I}, x_{I I}\right\}$ with the basis $\mathscr{B}_{T_{\mathbf{x}} \Omega}=\left\{\mathbf{i}_{I}, \mathbf{i}_{I I}\right\}$.

Let us define the angle $\beta$ (see Fig. 4 ) that $\mathbf{v}_{I}$ and $\mathbf{w}$ form in $T_{\Phi(\mathbf{X})} \Omega$. This angle can be characterized by

$$
\begin{aligned}
& \cos \beta=\frac{\mathbf{V}_{I} \cdot \mathbf{C E}}{\sqrt{\mathbf{V}_{I} \cdot \mathbf{C} \mathbf{V}_{I}} \sqrt{\mathbf{E} \cdot \mathbf{C E}}}, \\
& \sin \beta=\frac{\mathbf{V}_{I I} \cdot \mathbf{C E}}{\sqrt{\mathbf{V}_{I I} \cdot \mathbf{C V} \mathbf{V}_{I I}} \sqrt{\mathbf{E} \cdot \mathbf{C E}}} .
\end{aligned}
$$

The length of the tangent deformed lattic ${ }^{13}$ vector $\mathbf{w}$ can be obtained as

$$
\|\mathbf{w}\|=A \Lambda,
$$




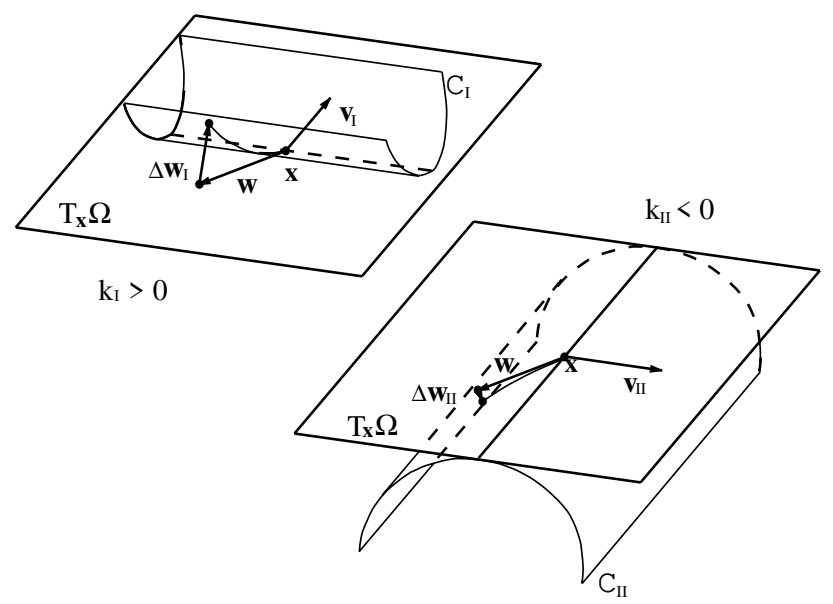

Fig. 5. Exponential map in fictitious cylinders in each principal direction.

where $\Lambda=\sqrt{\mathbf{E} \cdot \mathbf{C E}}$ is the stretch in the direction of the lattice vector. Consequently, the components of $\mathbf{w}$ in the basis $\mathscr{B}_{T_{\mathbf{x}} \Omega}$ are:

$$
\left\{\begin{array}{c}
w_{I} \\
w_{I I}
\end{array}\right\}=\left\{\begin{array}{c}
A \Lambda \cos \beta \\
A \Lambda \sin \beta
\end{array}\right\}=\left\{\begin{array}{c}
A \frac{\mathbf{V}_{I} \cdot \mathbf{C E}}{\sqrt{\mathbf{V}_{I} \cdot \mathbf{C} \mathbf{V}_{I}}} \\
A \frac{\mathbf{V}_{I I} \cdot \mathbf{C E}}{\sqrt{\mathbf{V}_{I I} \cdot \mathbf{C} \mathbf{V}_{I I}}}
\end{array}\right\}=\left\{\begin{array}{c}
A \frac{\left\langle\mathbf{V}_{I} \mid \mathbf{E}\right\rangle_{\mathbf{C}}}{\left\|\mathbf{V}_{I}\right\|_{\mathbf{C}}} \\
A \frac{\left\langle\mathbf{V}_{I I} \mid \mathbf{E}\right\rangle_{\mathbf{C}}}{\left\|\mathbf{V}_{I I}\right\|_{\mathbf{C}}}
\end{array}\right\} .
$$

\subsection{Principal direction $I$}

Fig. 5 illustrates the approximation to the exponential map for a surface with negative Gaussian curvature - a hyperbolic point. Consider a fictitious cylinder $\mathscr{C}_{I}$ of radius $1 / k_{I}$ passing through point $\mathbf{x}=\Phi(\mathbf{X})$ whose tangent plane is $T_{\mathbf{x}} \Omega$ and whose axis is perpendicular to $\mathbf{v}_{I}$.

The cylinder $\mathscr{C}_{I}$ can be parametrized isometrically from $T_{\mathbf{x}} \Omega$ into $\mathbb{R}^{3}$ as

$$
\mathscr{C}_{I}: \mathbf{f}_{I}\left(x_{I}, x_{I I}\right)=\left\{\begin{array}{c}
\frac{1}{k_{I}} \sin k_{I} x_{I} \\
x_{I I} \\
\frac{1}{k_{I}}\left(1-\cos k_{I} x_{I}\right)
\end{array}\right\} .
$$

The geodesic of this cylinder passing through $\mathbf{x}$ and tangent to $\mathbf{w}$ is

$$
\mathbf{c}(s)=\left\{\begin{array}{c}
\frac{1}{k_{I}} \sin \left[k_{I}(\cos \beta) s\right] \\
(\sin \beta) s \\
\frac{1}{k_{I}}\left(1-\cos \left[k_{I}(\cos \beta) s\right]\right)
\end{array}\right\}, 14
$$


where $s$ denotes the arc-length parameter. Consequently, by evaluating the above expression at $s=\|\mathbf{w}\|$, the image of $\mathbf{w}$ through exponential map of $\mathscr{C}_{I}$ at $\mathbf{x}$ is

$$
\left[\exp _{\mathbf{x}, I} \mathbf{w}\right]_{\mathscr{B}_{\mathbb{R}^{3}}}=\left\{\begin{array}{c}
\frac{1}{k_{I}} \sin k_{I} w_{I} \\
w_{I I} \\
\frac{1}{k_{I}}\left(1-\cos k_{I} w_{I}\right)
\end{array}\right\} .
$$

Finally, the exponential correction in the first principal direction, that is the difference between the above vector and $\mathbf{w}$, is

$$
\left[\Delta \mathbf{w}_{I}\right]_{\mathscr{B}_{\mathbb{R}^{3}}}=\left\{\begin{array}{c}
\frac{1}{k_{I}} \sin k_{I} w_{I}-w_{I} \\
0 \\
\frac{1}{k_{I}}\left(1-\cos k_{I} w_{I}\right)
\end{array}\right\} .
$$

\subsection{Principal direction $I I$}

In this case, the parametrization of the cylinder $\mathscr{C}_{I I}$ of radius $1 / k_{I I}$ in the coordinate systems described above is

$$
\mathscr{C}_{I I}: \mathbf{f}_{I I}\left(x_{I}, x_{I I}\right)=\left\{\begin{array}{c}
x_{I} \\
\frac{1}{k_{I I}} \sin k_{I I} x_{I I} \\
\frac{1}{k_{I I}}\left(1-\cos k_{I I} x_{I I}\right)
\end{array}\right\}
$$

and similarly we obtain

$$
\left[\Delta \mathbf{w}_{I I}\right]_{\mathscr{B}_{\mathbb{R}^{3}}}=\left\{\begin{array}{c}
0 \\
\frac{1}{k_{I I}} \sin k_{I I} w_{I I}-w_{I I} \\
\frac{1}{k_{I I}}\left(1-\cos k_{I I} w_{I I}\right)
\end{array}\right\} .
$$

Note that all the deformed geometric quantities are expressed in the undeformed configuration.

\subsection{Final formula}

The Exponential Born rule $\mathbf{a}=\exp _{\Phi(\mathbf{X})} \circ \mathbf{F A}$ is then approximated by the map $\mathbf{a}=$ $\mathbf{F A}+\Delta \mathbf{w}_{I}+\Delta \mathbf{w}_{I I}$. By defining $\mathscr{Z}(x)=\sin x / x$, the expression for the deformed lattice 
vector in the orthonormal basis $\mathscr{B}_{\mathbb{R}^{3}}$ is

$$
[\mathbf{a}]_{\mathscr{B}_{\mathbb{R}^{3}}}=\left\{\begin{array}{c}
w_{I} \mathscr{Q}\left(k_{I} w_{I}\right) \\
w_{I I} \mathscr{Q}\left(k_{I I} w_{I I}\right) \\
\frac{k_{I} w_{I}^{2}}{2} \mathscr{Q}^{2}\left(k_{I} w_{I} / 2\right)+\frac{k_{I I} w_{I I}^{2}}{2} \mathscr{Q}^{2}\left(k_{I I} w_{I I} / 2\right)
\end{array}\right\} .
$$

Bearing Eqs. (30) and (33) in mind, and the fact that $k_{I, I I}$ and $\mathbf{V}_{I, I I}$ are obtained from the eigenvalue problem (24), it is clear that $[\mathbf{a}]_{\mathscr{B}_{\mathbb{R}^{3}}}$ depends only on the undeformed lattice vector $\mathbf{A}$, the Green deformation tensor $\mathbf{C}$ and the pull-back of the curvature tensor $\mathscr{K}$. Deformed geometric quantities can readily be obtained from (40). We can symbolically write the length of a deformed lattice vector $a_{i}$ and the angle between two lattice vectors $\theta_{j k}$, in terms of the undeformed crystal and the local strain measures:

$$
a_{i}=f\left(\mathbf{C}, \mathscr{K} ; \mathbf{A}_{i}\right) \quad \text { and } \quad \theta_{j k}=g\left(\mathbf{C}, \mathscr{K} ; \mathbf{A}_{j}, \mathbf{A}_{k}\right) .
$$

Remark 4. In the numerical implementation, the function $\mathscr{2}(x)=\sin x / x$ should be treated carefully to avoid large round-off errors. Its limit when the argument tends to zero is one. Here, this function is evaluated by a Taylor expansion when $x$ is smaller than a certain threshold.

\section{Membrane formulation}

The present section describes the formulation of the continuum membrane mechanics. The membrane constitutive model based on the atomistic potential energy is outlined in Section 6.1. The essential ideas can also be found in other works dealing the quasicontinuum method for bulk materials (Tadmor et al., 1996; Shenoy et al., 1999; Tadmor et al., 1999). Then, Section 6.2 describes the formulation of the continuum counterpart of the long-range interactions. Finally, Section 6.3 gives the continuum variational problem as well as Lagrangian stress measures conjugate to the considered strain measures.

\subsection{Constitutive model}

So far, we have related the deformation of the atomic system to that of an equivalent continuum membrane. No particular assumption about the underlying atomic model has been made. The kinematic relation $\mathbf{a}=\mathscr{F}_{\Phi}(\mathbf{A})$ can be used to derive continuum hyper-elastic potentials for a wide variety of atomic models consistent with the Born-Oppenheimer approximation, i.e. where the energy can be obtained in terms of the nuclear positions. Thes $\mathbb{e}^{6}$ models include analytic empirical potentials and quantum-mechanical models such as tight-binding models, see e.g. Tadmor et al. (1999). 


\subsubsection{Atomic model}

In the following, a particular atomistic model is considered. Our interest here is to model covalently bonded systems, and it is assumed that the topology of the bond network does not change, i.e. there is no bond breaking or formation. For such a system, the energy can be expressed in terms of the bond geometry, i.e. their length and the angles between adjoining bonds:

$$
E=E\left(r_{i}, \theta_{j}\right)
$$

where $r_{i}$ denotes the lengths of the covalent bonds of the system and the $\theta_{j}$ the angles. One instance of such a model is the many-body expansion of the potential energy, for example a 2-body/3-body expansion:

$$
E=\sum_{i} \mathscr{V}_{s}\left(r_{i}\right)+\sum_{k} \mathscr{V}_{\theta}\left(\theta_{k}, r_{k}^{1}, r_{k}^{2}\right)
$$

where the first sum runs over the covalent bonds, the second over the angles formed by covalent bonds. Here, $r_{k}^{1}$ and $r_{k}^{2}$ denote the lengths of the bonds that form the angle $\theta_{k}$. The MM2/MM3 models fall within this formalism. Other examples are bond order potentials, such as those for hydrocarbons proposed by Brenner (1990):

$$
E=\sum_{i}\left[\mathscr{V}_{R}\left(r_{i}\right)-b_{i}\left(r_{i}^{j}, \theta_{i}^{j}\right) \mathscr{V}_{A}\left(r_{i}\right)\right]
$$

where the sum runs over the bonds, and the bond order function $b_{i}$ depends on the lengths of all the bonds connected to the $i$ th bond, $r_{i}^{j}$, and on the angles they form with the $i$ th bond, $\theta_{i}^{j}$.

Remark 5. The use of these atomic potentials for covalent systems, in which the potential energy at a nucleus or bond depends on the lengths and angles directly adjacent to it allows closed-form expressions for the continuum strain energy density to be obtained. Predominant short range interactions also support the use of a local continuum model. This contrasts with the situation in other works (Tadmor et al., 1996) where materials with longer range interactions are considered. In these cases, the continuum strain energy density is computed by local constrained atomistic calculations, and non-local effects need to be included.

Remark 6. In the literature dealing with the non-linear elasticity of crystals, an important concern is the implications of non-convexity and the presence of invariant groups in the strain energy. Non-convexity is responsible for many important phenomena such as the development of stable dislocations and phase changes, and the continuum variational problem may then not have a solution (see Dacorogna (1989, p. 276) and references therein). In the present work, these issues are not dealt with.

\subsubsection{Simple Bravais lattices}

Consider that the undeformed planar crystal is a simple Bravais lattice, i.e. the atomic positions $\mathbf{X}_{n}$ can be defined relative to an atomic site as follows:

$$
\mathbf{X}_{n}=n^{i} \mathbf{B}_{i}, \quad i=1,2,
$$


where $\mathbf{B}_{i}$ denote the Bravais basis vectors and $n^{i}$ are integers. Therefore, the covalent bonds $\mathbf{A}_{k}$ are integer linear combinations of the basis vectors of the crystal, $\mathbf{A}_{k}=n_{k}^{i} \mathbf{B}_{i}$, with $n_{k}^{i}$ being typically 0 or 1 . Using the approximation to the Exponential Born rule described in the previous section, we can obtain deformed bond lengths and angles in terms of the continuum deformation. Plugging Eqs. (41) into Eq. (42), and considering a representative cell of the crystal containing all energetically relevant bonds and angles - a representative volume element, RVE-, we can write the continuum hyper-elastic potential as

$$
W(\mathbf{C}, \mathscr{K})=\frac{1}{S_{0}} E_{\mathrm{Cell}}\left(a_{i}, \theta_{j k}\right),
$$

where $S_{0}$ denotes the area of the undeformed representative cell. Although not explicitly shown, this hyper-elastic potential also depends on the undeformed crystal structure, and inherits its symmetries. Moreover, this model is automatically frame indifferent.

\subsubsection{Bravais multi-lattices}

A Bravais multi-lattice can be seen as a collection of interpenetrating simple Bravais lattices, where the atomic sites can be obtained by

$$
\mathbf{X}_{n}=n^{i} \mathbf{B}_{i}+\mathbf{P}_{k}, \quad i=1,2,
$$

where, as before, $\mathbf{B}_{i}$ denote the basis or lattice vectors and $\mathbf{P}_{k}, k=1, \ldots, K$ represent shifts of the different simple lattices. In such a lattice, in order to generate all the atomic positions, $K+1$ basis nuclei are needed in addition to the basis vectors (in a simple Bravais lattice, $K=0$, only one primitive atom is needed). The honeycomb structure of the graphene sheets (see Fig. 6) is an example of a multi-lattice. Note that one nuclear site (say a black one) and the basis vectors $\mathbf{B}_{1}$ and $\mathbf{B}_{2}$ are not enough to construct the entire lattice, in particular the white sites. Either a white site or the shift vector $\mathbf{P}$ is also needed.

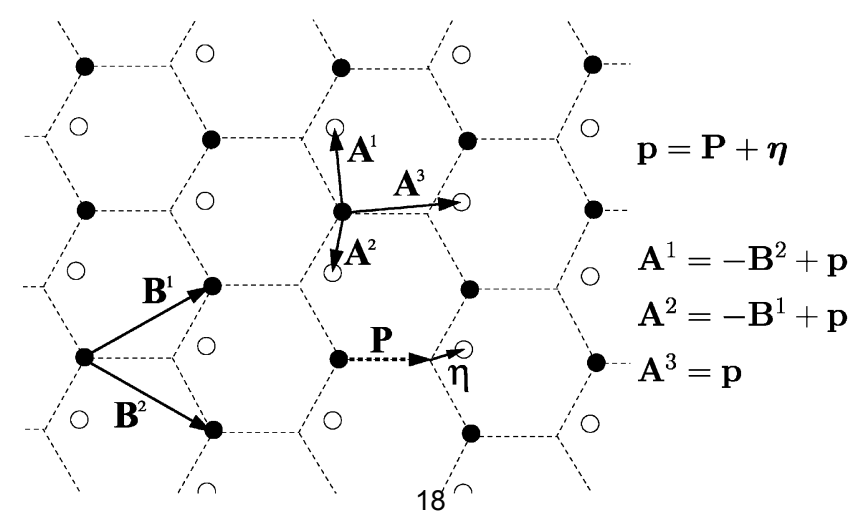

Fig. 6. Honeycomb Bravais multi-lattice: basis vectors $\mathbf{B}_{i}$, shift vector $\mathbf{P}$, inner displacements $\boldsymbol{\eta}$ and bond vectors $\mathbf{A}_{k}$. 
In classical crystal elasticity, the Born rule is postulated to affect the basis vectors, while the evolution of the shift vectors is viewed as an internal rearrangement (Cousins, 1978; Zanzotto, 1996). This microstructure is obtained by minimizing the energy with respect to the shift vectors, for each given macroscopic continuum deformation. Following Tadmor et al. (1999), the inner displacements $\boldsymbol{\eta}_{k}$ are defined in the reference crystal. Therefore, a bond vector, defined as a linear combination of basis and shifts vectors, $\mathbf{A}^{0}=n^{i} \mathbf{B}_{i}+m^{k} \mathbf{P}_{k}$, is first transformed due to the internal rearrangement into $\mathbf{A}=n^{i} \mathbf{B}_{i}+m^{k}\left(\mathbf{P}_{k}+\boldsymbol{\eta}_{k}\right)$, and then transformed according to the Born rule by

$$
\mathbf{a}=\mathbf{F}\left(n^{i} \mathbf{B}_{i}+m^{k}\left(\mathbf{P}_{k}+\boldsymbol{\eta}_{k}\right)\right) \text {. }
$$

In the present membrane theory, we will include the effect of the inner displacements simply by rewriting Eq. (48) as follows:

$$
\mathbf{a}=\exp _{\Phi(\mathbf{X})} \circ \mathbf{F}(\mathbf{X})\left(n^{i} \mathbf{B}_{i}+m^{k}\left(\mathbf{P}_{k}+\boldsymbol{\eta}_{k}\right)\right) .
$$

Note that by doing this, we only allow for inner rearrangements in the original planar lattice. Therefore, the inner displacements are viewed as an "in plane" effect and the local rearrangements of the basis atoms cannot move atoms "out of the surface".

If the approximation to the Exponential Born rule described in Section 5 is adopted, we have

$$
W=W\left(\mathbf{C}, \mathscr{K}, \boldsymbol{\eta}_{k}\right)
$$

Thus, the continuum strain energy density depends not only on the local deformation of the membrane, but also on the internal variables $\boldsymbol{\eta}_{k}$. Then, given a local deformation, the energy is minimized with respect to inner displacements:

$$
\hat{\boldsymbol{\eta}}_{k}(\mathbf{C}, \mathscr{K})=\left.\arg \left(\min _{\boldsymbol{\eta}_{k}} W\left(\mathbf{C}, \mathscr{K}, \boldsymbol{\eta}_{k}\right)\right) \Rightarrow \frac{\partial W}{\partial \boldsymbol{\eta}_{k}}\right|_{\hat{\boldsymbol{\eta}}_{k}}=\mathbf{0} .
$$

After this internal relaxation, the continuum strain energy density can be written exclusively in terms of the local continuum deformation:

$$
W=W\left(\mathbf{C}, \mathscr{K}, \hat{\boldsymbol{\eta}}_{k}(\mathbf{C}, \mathscr{K})\right)=\hat{W}(\mathbf{C}, \mathscr{K}) .
$$

Fig. 6 illustrates some of these ideas. The dashed lines denote the original bond network. Then, the shift vector is perturbed by an inner displacement $\boldsymbol{\eta}$, translating the white nuclei with respect to the black ones. Note that the Bravais basis vectors that allow the generation of nuclear positions are unchanged, yet the microstructure (relative arrangement of the basis sites) as well as the bond vectors $\mathbf{A}_{i}$ are changed, and so is the energy of the crystal. Then, a macroscopic deformation can be superimposed to this microstructural change.

As noted by Tadmor et al. (1999), the derivatives of the resulting strain energy density with respect to the strain measures, for instance $\mathbf{C}$, can be computed as

$$
\frac{\partial \hat{W}}{\partial \mathbf{C}}=\frac{\partial W}{\partial \mathbf{C}}+\frac{\partial W}{\partial \eta_{k}^{i}} \frac{\partial \hat{\eta}_{k}^{i}}{\partial \mathbf{C}}
$$


But, using the fact that when evaluated at the relaxed inner displacements the second part of Eq. (51) holds, we have the simpler expression,

$$
\frac{\partial \hat{W}}{\partial \mathbf{C}}=\left.\frac{\partial W}{\partial \mathbf{C}}\right|_{\hat{\boldsymbol{\eta}}_{k}} \text {. }
$$

The same holds for the derivatives of the relaxed strain energy $\hat{W}$ with respect to the curvature.

\subsection{Non-bonded interactions and body forces}

The non-bonded or van der Waals interactions include the attractive London dispersion forces as well as the overlap repulsion forces. In the $\mathrm{MD} / \mathrm{MM}$ literature, these interactions are accounted for using inter-atomic potentials that only act between non-bonded pairs of atoms. This diffuse interactions are critical in many applications. For the molecular system we have for instance

$$
\mathscr{V}_{\text {tot, } \mathrm{nb}}=\sum_{i} \sum_{j>i, j \notin B_{i}} \mathscr{V}_{\mathrm{nb}}\left(r_{i j}\right)=\frac{1}{2} \sum_{i} \sum_{j \notin B_{i}} \mathscr{V}_{\mathrm{nb}}\left(r_{i j}\right),
$$

where $\mathscr{V}_{\mathrm{nb}}$ is the non-bonded potential and $B_{i}$ is the set of atoms bonded to atom $i$. The second version of the total non-bonded potential is provided because it makes easier the continuum formulation.

A simple argument involving two RVE's of area $S_{0}$ containing two nuclei each allows to write the continuum counterpart as

$$
\Pi_{\mathrm{nb}}=\frac{1}{2} \frac{4}{S_{0}^{2}} \int_{\Omega_{0}} \int_{\Omega_{0}-B_{\mathbf{X}}} \mathscr{V}_{\mathrm{nb}}(\|\Phi(\mathbf{X})-\Phi(\mathbf{Y})\|) \mathrm{d} \Omega_{0 Y} \mathrm{~d} \Omega_{0 X},
$$

where $B_{\mathbf{X}}$ is a ball centered at $\mathbf{X}$ and with a radius which is function of the undeformed bond length to account for the fact that this potential does not affect bonded atoms. If this non-bonded potential term is evaluated using numerical quadrature, we have

$$
\begin{aligned}
\Pi_{\mathrm{nb}} & =\frac{1}{2} \frac{4}{S_{0}^{2}} \sum_{i} \sum_{j \notin B_{i}} \mathscr{V}_{\mathrm{nb}}\left(\left\|\Phi\left(\mathbf{X}_{i}\right)-\Phi\left(\mathbf{X}_{j}\right)\right\|\right) w_{i} w_{j} \\
& =\frac{4}{S_{0}^{2}} \sum_{i} \sum_{j>i, j \notin B_{i}} \mathscr{V}_{\mathrm{nb}}\left(\left\|\Phi\left(\mathbf{X}_{i}\right)-\Phi\left(\mathbf{X}_{j}\right)\right\|\right) w_{i} w_{j},
\end{aligned}
$$

where $w_{i}$ denote the integration weights and $\mathbf{X}_{i}$ the sample points. Note that with the numerical integration, the structure of the original potential, which loops over interactions and not twice over the domain, is recovered.

When external forces are applied on the nuclei (e.g. electrostatic forces), the continuum counterpart is a body force, and the corresponding exterior potential is

$$
\Pi_{\mathrm{ext}}=\int_{\Omega_{0}} \mathbf{B} \cdot \Phi \mathrm{d} \Omega_{0},
$$

where $\mathbf{B}$ is the body force per unit undeformed area. If the forces applied on the atomic system are a certain constant force $\mathbf{f}$ acting on each atom, then $\mathbf{B}$ is simply given by 
the expression

$$
\mathbf{B}=\frac{2}{S_{0}} \mathbf{f}
$$

\subsection{Variational principle and stress measures}

The total potential energy of a given deformation map $\Psi$ is

$$
\begin{aligned}
\Pi(\Psi)= & \Pi_{\mathrm{int}}-\Pi_{\mathrm{ext}}+\Pi_{\mathrm{nb}} \\
= & \int_{\Omega_{0}} \hat{W}(\mathbf{C}(\Psi), \mathscr{K}(\Psi)) \mathrm{d} \Omega_{0}-\int_{\Omega_{0}} \mathbf{B} \cdot \Psi \mathrm{d} \Omega_{0} \\
& +\frac{1}{2} \frac{4}{S_{0}^{2}} \int_{\Omega_{0}} \int_{\Omega_{0}-B_{\mathbf{X}}} \mathscr{V}_{\mathrm{nb}}(\|\Psi(\mathbf{X})-\Psi(\mathbf{Y})\|) \mathrm{d} \Omega_{0 Y} \mathrm{~d} \Omega_{0 X} .
\end{aligned}
$$

The above includes the bonded potentials, non-bonded interactions and body forces, and assumes that the approximation of the exponential map leads to a local strain energy density that depends on $\mathbf{C}$ and $\mathscr{K}$. The equilibrium deformation maps of the system are then given by

$$
\Phi=\arg \left[\inf _{\Psi \in \mathscr{C}} \Pi(\Psi)\right],
$$

where $\mathscr{C}$ is the appropriate space of deformation maps or trial functions accounting for essential boundary conditions. According to the principle of stationary energy, the equilibrium configurations of the system $\Phi$ are stationary points of the potential energy functional, and therefore verify

$$
0=\delta \Pi(\Phi)=\int_{\Omega_{0}}\left(\frac{\partial \hat{W}}{\partial \mathbf{C}}: \delta \mathbf{C}+\frac{\partial \hat{W}}{\partial \mathscr{K}}: \delta \mathscr{K}\right) \mathrm{d} \Omega_{0}-\int_{\Omega_{0}} \mathbf{B} \cdot \delta \Phi \mathrm{d} \Omega_{0}+\delta \Pi_{\mathrm{nb}},
$$

where the variations $\delta \Phi$ lie in a corresponding space $\mathscr{V}$. The variations of the nonbonded continuum potential can be written as

$$
\begin{aligned}
\delta \Pi_{\mathrm{nb}}= & \frac{1}{2} \frac{4}{S_{0}^{2}} \int_{\Omega_{0}} \int_{\Omega_{0}-B_{\mathbf{X}}} \frac{\mathscr{V}_{\mathrm{nb}}^{\prime}}{\|\Phi(\mathbf{X})-\Phi(\mathbf{Y})\|}[\Phi(\mathbf{X})-\Phi(\mathbf{Y})] \\
& \cdot[\delta \Phi(\mathbf{X})-\delta \Phi(\mathbf{Y})] \mathrm{d} \Omega_{0 Y} \mathrm{~d} \Omega_{0 X} .
\end{aligned}
$$

The stress measures are evaluated at the relaxed inner displacements $\hat{\boldsymbol{\eta}}$ and are given by

$$
\mathbf{n}=2 \frac{\partial \hat{W}}{\partial \mathbf{C}}=2 \frac{\partial W}{\partial \mathbf{C}} \quad \text { and } \quad \mathbf{m}=\frac{\partial \hat{W}}{\partial \mathscr{K}}=\frac{\partial W}{\partial \mathscr{K}},
$$

following a similar rationale to that used to obtain Eq. (54). The first of these stress measures corresponds to the Second Piæla-Kirchhoff stress, while the second is a moment-like stress. These stress tensors, when contracted with the appropriate normal to a curve in the undeformed body, provide tractions expressed as force per unit length 
and moment per unit length, respectively. Unlike classical shell theory, the stress tensors are not stress resultants, because the body is intrinsically a two-manifold without thickness, rather than a bulk material with additional kinematic constraints.

Using the Green strain tensor $\mathbf{E}=1 / 2(\mathbf{C}-\mathbf{I})$, we can rewrite the principle of virtual work as

$$
0=\int_{\Omega_{0}}(\mathbf{n}: \delta \mathbf{E}+\mathbf{m}: \delta \mathscr{K}) \mathrm{d} \Omega_{0}-\int_{\Omega_{0}} \mathbf{B} \cdot \delta \Phi \mathrm{d} \Omega_{0}+\delta \Pi_{\mathrm{nb}} .
$$

\section{Numerical simulations of carbon nanotubes}

In this section, the mechanics of carbon nanotubes are studied with the developed hyper-elastic membrane. The appendix provides details about the implementation of the model for carbon nanotubes for a 2-body/3-body potential. The two-body potential $\mathscr{V}_{S}$ is a Morse potential while the three-body potential $\mathscr{V}_{\theta}$ depends only on the angle and is harmonic with a sextic correction. The parameters are taken from the MM2 model.

In all the simulations, the inner relaxation is performed by Newton's method. For the problem at hand, typical over-estimates of about $15 \%$ in the equilibrium energy and noticeably different equilibrium configurations are observed in the absence of the inner lattice relaxation.

Equilibrium configurations are obtained by minimization of the total potential energy of the continuum system using the Conjugate Gradient method (Gilbert and Nocedal, 1992). These are compared to MM simulations of the atomistic counterpart. The variational principle described in Eq. (65) is discretized by Galerkin finite elements (FE). Thus, the original discrete molecular system is replaced by a continuum model which is subsequently transformed by the FE method into another discrete system. However, we are free to construct the FE discretization so that the FE model has far fewer degrees-of-freedom than the original system.

The variational principle (65) imposes restrictions on the finite element approximation spaces. The virtual internal work term involves variations of the curvature of the test functions, and therefore the finite element space needs to be $H^{2}$ (i.e. possess square integrable second derivatives). For approximation of the deformation, subdivision surfaces based on Loop's scheme are used. This technique originates from the field of computational geometry and has recently been applied to thin shell analysis (Cirak et al., 2000; Cirak and Ortiz, 2001). In this method, a control mesh of triangular elements with only translational degrees-of-freedom is used to construct a smooth $\left(H^{2}\right)$ surface. Very simple recursive subdivision rules applied on the control mesh are shown to lead to smooth surfaces. What makes the technique applicable to thin-shell analysis is the fact that the limiting process is not required in order to obtain the limit surface. A closed-form parametrization of the surface can easily be constructed, and therefore the strain measures can be computed.

In the presented simulations, nanotubes22re twisted and bent beyond structural instabilities, after which buckled configurations have lower energy than their homogeneous counterparts. In the case of bending, a characteristic kink or local buckle is observed. 
There is experimental evidence (Chopra et al., 1995; Bernholc et al., 1998; Falvo et al., 1997) of the existence of such buckled configurations.

The numerical experiments are carried out by incrementally rotating with opposing angles both ends of the nanotube. For the twisting computations, the rotation axis coincides with that of the nanotube, while in the bending experiments the ends are rotated with respect to the axis perpendicular to the axis of the nanotube through its center. The twisting or bending angle is measured as the total rotation angle that each end of the nanotube undergoes.

\subsection{Twisting of a [10-10] nanotube}

In our studies of twisting, we consider a [10-10] nanotube (the standard description of carbon nanotubes and their chirality in terms of two integers is described in Saito et al., 1992 ) about $12 \mathrm{~nm}$ long (50 hexagonal cells in its length). Note that the chirality of the nanotube is naturally accounted for in the membrane model through the orientation of the underlying crystal with respect to the rolling direction used to form the nanotube. The total twisting angle is $25^{\circ}$ on each end. The rotation angle increments are of $1^{\circ}$ for the finite element membrane and of $0.5^{\circ}$ for the atomistic system. Smaller increments are required in the latter for convergence of the minimization procedure. The atomistic system has 2020 nuclei while the finite element model 910 nodes. This example is intended to provide a validation of the hyper-elastic membrane based on the Exponential Born rule in a large deformation situation. In addition, the same problem is solved for a membrane based on the standard Born rule to illustrate the pitfalls of this approach for surfaces.

Fig. 7 shows the evolution of the strain energy relative to the relaxed tube versus the twisting angle for both the atomistic model and the continuum membrane. The energy is expressed in atto Joules $\left(1 \mathrm{aJ}=10^{-18} \mathrm{~J}\right)$. The figure shows a first regime in which the energy grows roughly quadratically with deformation. This corresponds to a nearly homogeneous torsion of the tube. In this regime, the agreement between the two models is almost perfect. Then, at a twisting angle of about $9.5^{\circ}$, a structural instability occurs and the nanotube buckles releasing part of its stored deformation energy. After this point, the growth of the energy is roughly linear. It should be pointed out that an imperfection is introduced in the continuum membrane model. The unperturbed membrane buckles later, for a twisting angle of around $15^{\circ}$.

In the second regime, the agreement between the two models is also excellent, although discrepancies are noticeable. The maximum relative error in the energy is $2.4 \%$ for the largest angle. This error is due both to modeling and finite element discretization errors. In order to evaluate the contribution due to modeling approximations, the problem was solved with increasingly finer meshes until convergence was observed. The asymptotic value of the relative error in energy was found to be $1.9 \%$.

In Fig. 8a, the deformed configuration of the atomistic model for a twisting angle of $25^{\circ}$ is super-imposed onto the deformed continuum membrane. Both models agree remarkably well: the membrane nearly fit 23 he nuclear positions, despite the very large deformations. Fig. $8 \mathrm{~b}$ shows the strain energy density on the finite element computational mesh. It can be observed that highly deformed areas display high strain energy 


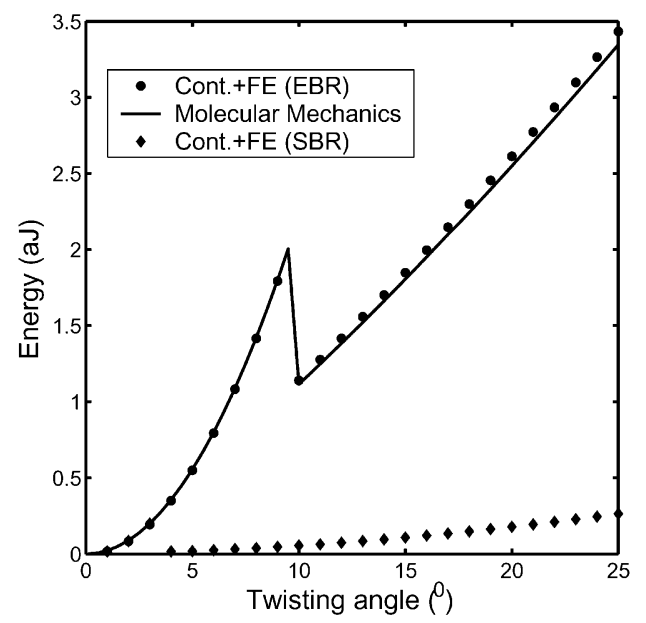

Fig. 7. Twisted [10-10] nanotube: comparison of the strain energy relative to the relaxed nanotube as a function of the twisting angle for molecular mechanics (line), the continuum membrane based on the Exponential Born rule (EBR, circles) and a continuum membrane based on the standard Born rule (SBR, diamonds).

density. This figure also illustrates the fact that the resolution of the finite element system is not dictated by the underlying crystal spacing, but can rather be adjusted depending on the deformation. In this example, the finite element simulations were one order of magnitude faster than the atomistic ones.

We next consider a membrane constructed from the standard Born rule without the proposed exponential extension. In this case the resulting hyper-elastic potential is non-convex. Indeed, as indicated in Section 2.2, the energy of such a model is invariant under isometric deformations, i.e. the model has zero bending stiffness. This causes a severe mesh dependence in the numerical solutions: since the discrete FE space cannot represent all isometric deformations, the discrete problem is still solvable, but as the mesh is refined, the numerical method provides solutions with increasingly fine features. Fig. 7 shows the strain energy evolution for this deficient model. It can be observed that energy predictions are adequate in the first few steps because for this particular example the homogeneous twisting solution is membrane dominant. However, a spurious instability occurs very early in the computation and the predicted energy is one order of magnitude smaller than the correct energy. Actually, if the boundary conditions were compatible with an isometric deformation, the predicted energy would be nearly zero, as shown in Arroyo and Belytschko (2001). Fig. 8c shows the equilibrium configuration for this model. It clearly differs both from the atomistic and the Exponential Born rule models, displaying unrealistic fine features.

\subsection{Bending of a [5-5] nanotube}

We consider a [5-5] carbon nanotube about $4.7 \mathrm{~nm}$ long (19.5 hexagonal cells in length). The tube is bent to an angle of $30^{\circ}$ at each end. This nanotube was chosen 


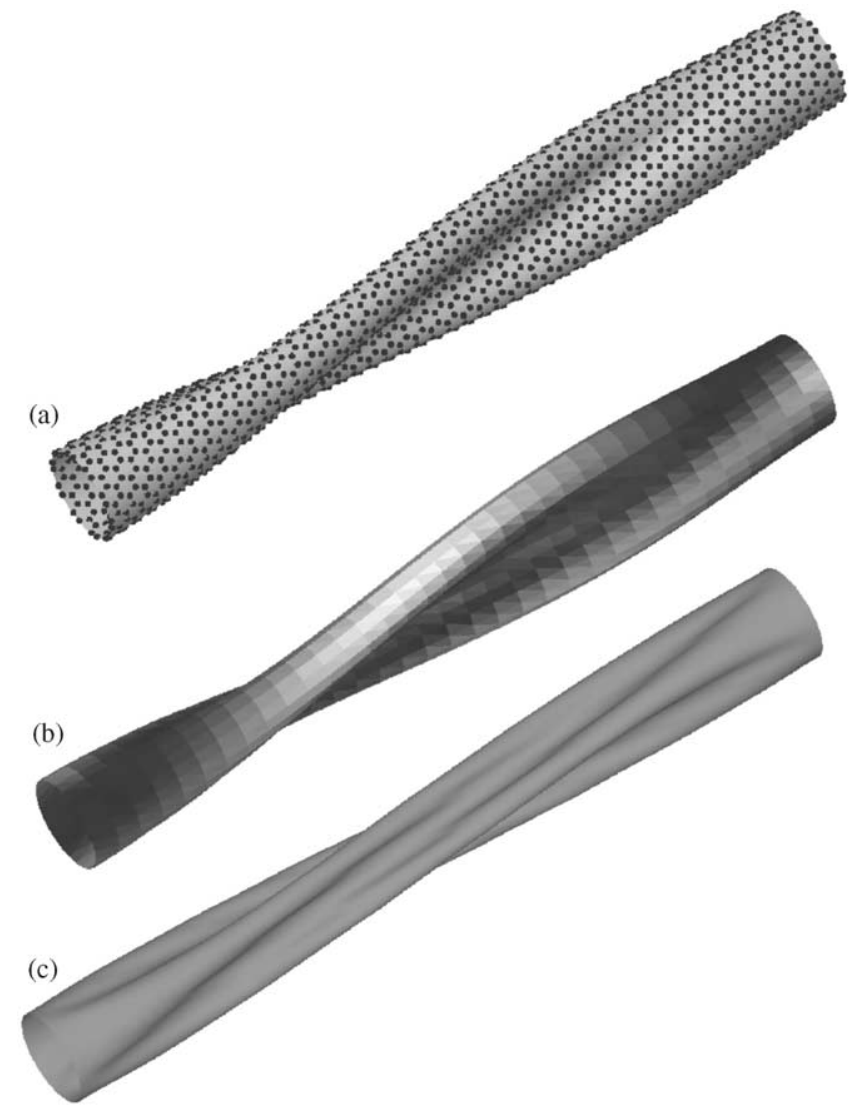

Fig. 8. Twisted [10-10] nanotube: (a) super-imposed MM (black spheres) and exponential Born rule based continuum membrane (gray surface) deformed configurations at a twisting angle of $25^{\circ}$; (b) map of the strain energy density (clear gray represents high strain energy density) for the finite element solution, illustrating the computational mesh and (c) deformed configuration for a membrane based on the standard Born rule.

because it poses a particularly stringent test to the continuum membrane model: it has only a few atoms around the circumference and very localized deformations occur. The limits of applicability of the membrane are thus explored. This example however does not exploit the computational savings that the continuum model can provide compared with full atomistic simulations.

Fig. 9 compares the deformation of the continuum membrane based on the Exponential Born rule to that of the atomistic model. The molecular mechanics results are displayed with spheres and segments representing atomic sites and covalent bonds, while the membrane results are post-processed as a translucent smooth surface. The qualitative agreement is very good. Before the $36 \mathrm{ckle}$ forms, at an angle of $10^{\circ}$, the atomic sites lie on the surface almost exactly. Then, as the buckle develops, the deformed geometries diverge slightly. However, even for very severe and localized deformations, 

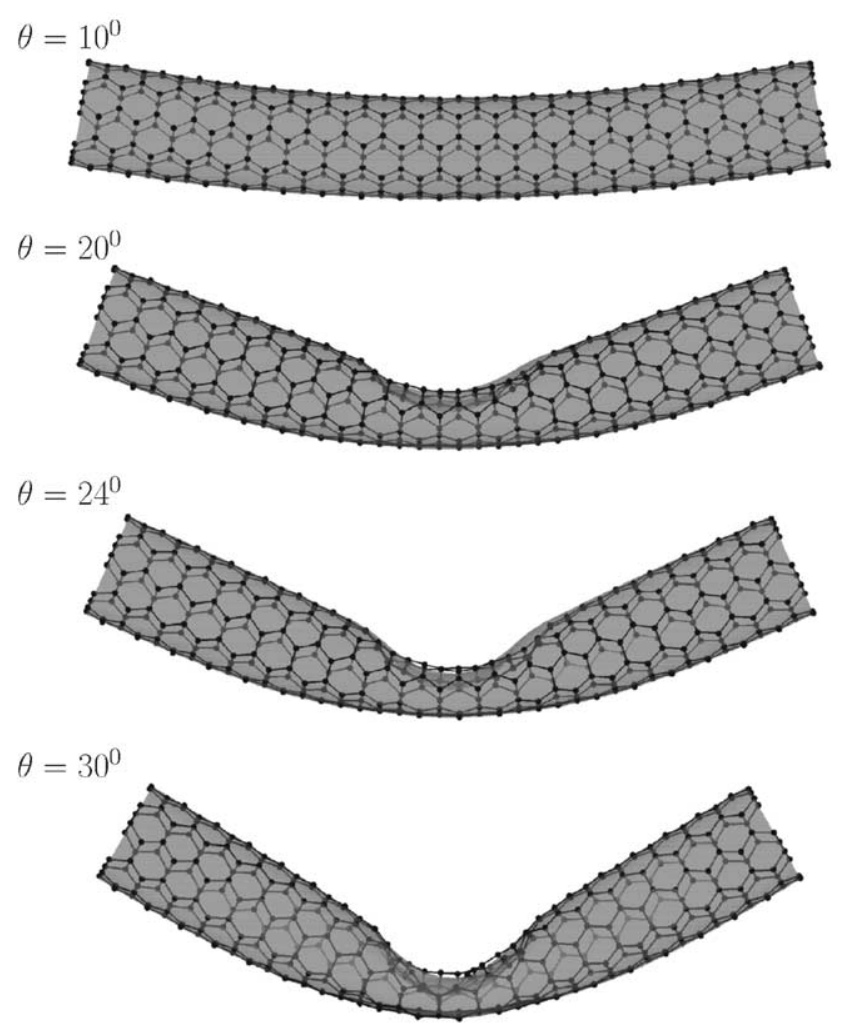

Fig. 9. Bent [5-5] nanotube: super-imposed MM (spheres and segments) and continuum membrane (translucent surface) deformed configurations.

the shape of the buckle is predicted remarkably well. Actually, the discrepancy can be partially explained by the fact that this nanotube is not symmetric while the buckle is symmetric. Therefore, in the molecular mechanics simulation the buckle does not appear in the center of the nanotube. The continuum membrane model cannot capture this asymmetric molecular arrangement and produces a buckle in the center of the nanotube.

Fig. 10 shows the evolution of the strain energy plotted against the bending angle for both models. The energies obtained with both models are undistinguishable until buckling occurs. Before this happens, the difference in energy between the two models is below $0.15 \%$. When the tube kinks, the energy evolution changes roughly from quadratic to linear. The angle at which the tube buckles is well predicted (around $11^{\circ}$ ). After this point, there is some discrepancy while the buckle fully develops. The larger difference in energy is $5.5 \%$ for an angle of $13^{\circ}$. Once the buckle has fully formed, the prediction of the membrane becomes excel 6 ent again, until the angle increases beyond $24^{\circ}$. At this point, a local energy release mechanism develops in the atomistic model. This mechanism is illustrated in Fig. 11, a zoom in on the buckle at angles of $24^{\circ}$ 


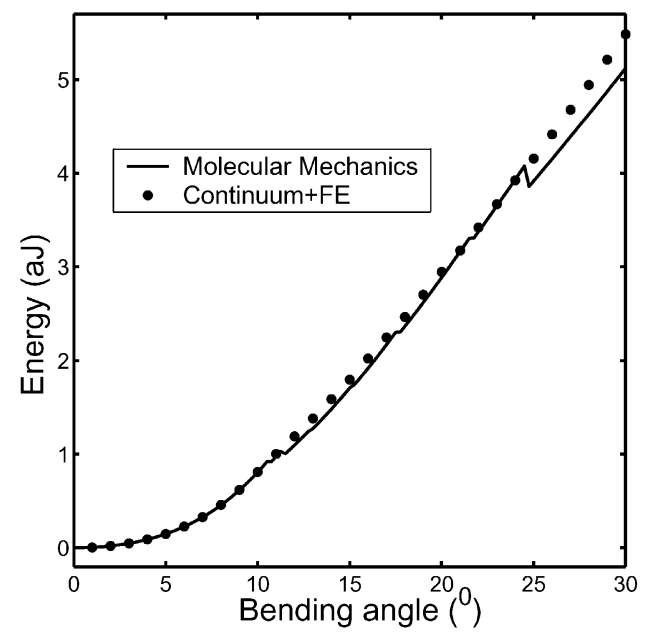

Fig. 10. Bent [5-5] nanotube: comparison of the strain energy relative to the relaxed nanotube for MM and the continuum membrane as a function of the bending angle.
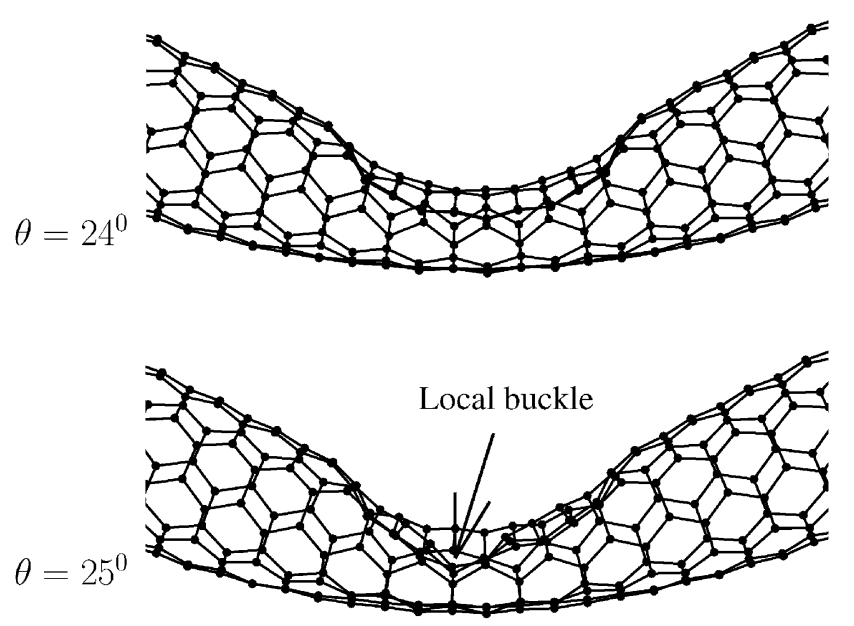

Fig. 11. Zoom in of the buckle: a local instability spanning two atomic spacings.

and $25^{\circ}$. A secondary buckle spanning only two bonds develops inside the kinked area. Such fine atomistic features are not captured by the continuum membrane model, and this explains the discrepancy in the energy for angles $>24^{\circ}$. Although this local energy release mechanism of the atomistie 27 system was not observed in all the bending experiments performed on [5-5] nanotubes, it illustrates the limitations of the membrane model in some situations. 


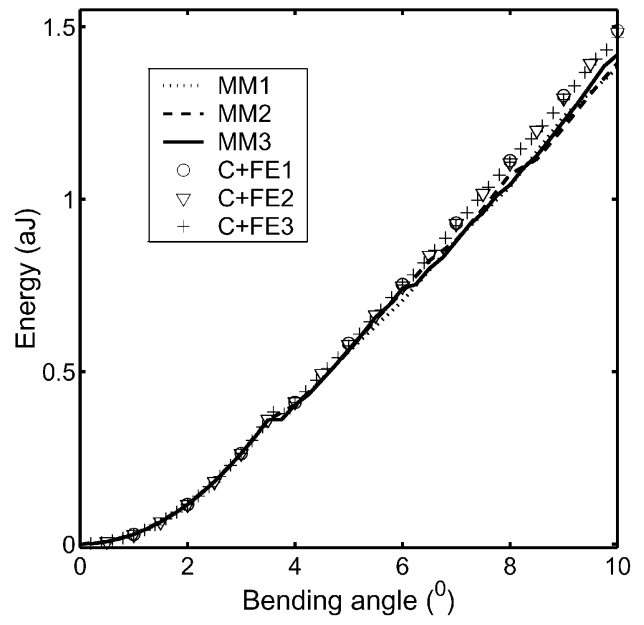

Fig. 12. Bent [10-10] nanotube: comparison of the strain energy relative to the relaxed nanotube for several $\mathrm{MM}$ and continuum simulations as a function of the bending angle.

\subsection{Bending of a [10-10] nanotube}

The last example considered is the bending of [10-10] nanotubes, 40 hexagonal cells in length. This nanotube is relatively more compliant than the [5-5] nanotube, and for a similar aspect ratio, the buckle forms at earlier stages. The results for three continuum membrane simulations with different load step-sizes are reported. Similarly, three atomistic simulations with different bending angle increments are considered.

Fig. 12 shows the energy growth with loading for the three molecular models (MM1, MM2 and MM3) as well as for the finite element membrane models $(\mathrm{C}+\mathrm{FE} 1, \mathrm{C}+\mathrm{FE} 2$ and $\mathrm{C}+\mathrm{FE} 3$ ). The six curves are indistinguishable until the buckling point. After this moment, they all remain very close. The energy differences between the continuum membrane simulations are within $1 \%$, while the atomistic simulations differ $<2.5 \%$. The maximum differences between all six models is below $4.5 \%$, and at the final stages the energy of the atomistic models is noticeably lower than that of the finite element membrane models.

Fig. 13 shows the final deformed configuration for the different continuum membrane models, while the deformed atomistic models MM1, MM2 and MM3 are displayed in Fig. 14. These figures show a wide variety of buckling configurations. The continuum membrane follows different equilibrium paths depending on the perturbations introduced by the numerical method (no deliberate perturbations were introduced). Either two or three buckles are observed. Note that these configurations are almost identical from the energetic point of view. This bending test is very sensitive to perturbations because a large portion of the nanotube reaches a critical compressive stress state at the onset of the instability, allowing for multiple Backling patterns with similar energetics. A similar situation is observed for the atomistic simulations that display different buckling patterns. 


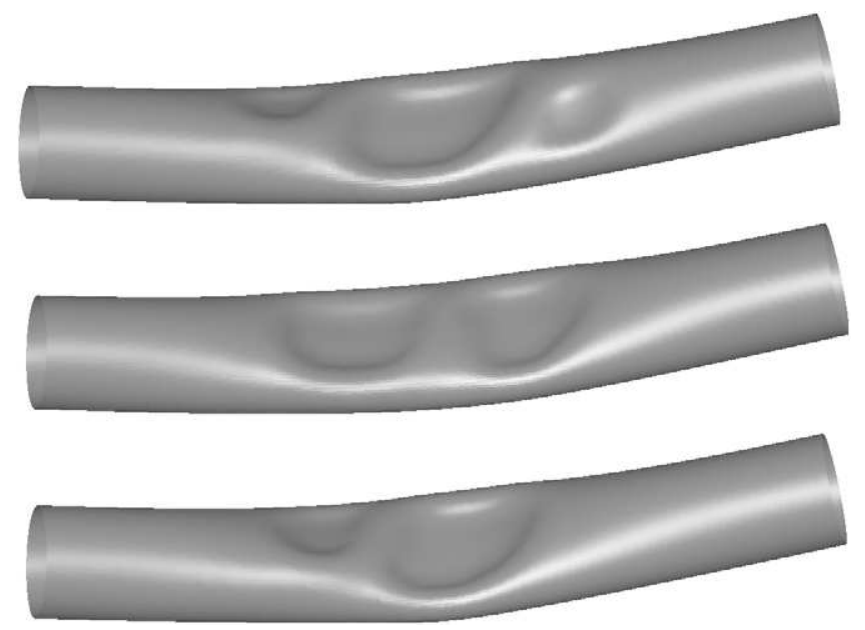

Fig. 13. Bent [10-10] nanotube: deformed configurations for three finite element simulations $(\mathrm{C}+\mathrm{FE} 1, \mathrm{C}+\mathrm{FE} 2$ and $\mathrm{C}+\mathrm{FE} 3$ from top to bottom).
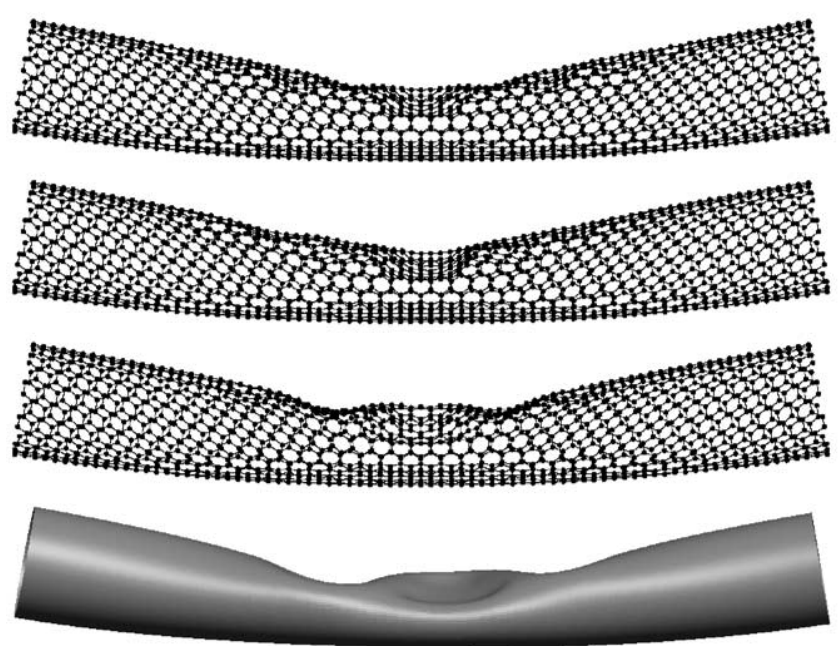

Fig. 14. Bent [10-10] nanotube: deformed configurations for three molecular mechanics simulations (MM1, MM2 and MM3 from top to bottom) and for finite element simulation C+FE4.

This issue is further investigated with a fourth continuum membrane simulation, $\mathrm{C}+\mathrm{FE} 4$. In this case, the initial guess at each loading stage is perturbed randomly. By doing this, we are able to access lower energy paths, and we actually obtain a deformed configuration with a very similar buckling 39ructure to that of atomistic simulation MM3 (see Fig. 14), with a central buckle facing the observer, and two lateral buckles facing the other side of the nanotube. By perturbing the continuum membrane in this fashion, 
we also come closer to simulation MM3 from the energetic point of view, with a relative error of only $1.5 \%$.

\section{Summary and discussion}

A methodology to construct continuum models for crystalline sheets of atoms has been presented. The proposed model is a hyper-elastic membrane whose material properties depend exclusively on the atomistic description of the system. The major contribution is an extension of Born rule to the case in which the crystal is not space-filling but rather a curved film. This extension is based on the exponential map. We have shown by physical arguments and numerical results that in the absence of the exponential map, the behavior of a continuum membrane does not reproduce the molecular mechanics model.

The exponential map is approximated locally to derive practical models. The strain energy of the membrane depends on the Green deformation tensor and on the pull-back of the curvature tensor, and is frame-indifferent. This elastic potential depends on these strain measures in a non-linear fashion that derives naturally from the Exponential Born rule. The membrane has no thickness. This contrasts with the usual elastic shell models found in the literature that assign a thickness to the crystalline film. In addition, a continuum formulation of the non-bonded interactions is derived.

We have used molecular mechanics solutions for carbon nanotubes as a benchmark since our primary aim was to mimic such discrete models with a continuum membrane. The continuum is discretized with subdivision finite elements, providing a smooth representation of the surface. Twisting, bending deformations of carbon nanotubes have been tested and excellent agreement between the two models has been observed even in the case of small nanotubes and very large deformations. Both the deformed configurations and the equilibrium energies were compared, and in most cases the agreement was excellent.

This continuum/finite element approach has advantages over atomistic simulations in a wide range of applications. The computational cost of the simulations can be dramatically reduced for wide and long nanotubes, multi-walled nanotubes and nanoropes. The continuum approach can also serve as an inexpensive way to obtain initial estimates of optimized structures to be refined with more sophisticated atomistic methods.

We have also explored the range of validity of the continuum membrane model. The twisting example illustrates that the atomistic system is more sensitive to buckling than the continuum membrane model. This is not unexpected, since the continuum membrane model cannot represent displacement fields with mode lengths of the order of the atomic spacing unless the finite element mesh is comparably refined. Furthermore, the behavior of cylindrical configurations is particularly susceptible to imperfections and perturbations, since these configurations are replete of symmetry-breaking modes. These phenomena are also illustrated by the bending example of [10-10] nanotubes, which displays energetically very close eâqilibrium paths with different buckling patterns. In the bending simulations of the [5-5] nanotube, we observed the inability of the continuum membrane to mimic a local energy release mechanism spanning two atomic 
spacings encountered in the atomistic system. Although these results are illustrative, the range of validity of this continuum membrane model needs to be investigated in more precise terms. In particular, it would be desirable to quantify the errors introduced by the approximation of the exponential map. Obviously, phenomena such as bond realignment, bond breaking and dislocations render the model in its present form inapplicable. However, we have demonstrated that the model is robust and accurate over a useful range of situations.

\section{Acknowledgements}

The support of the Office of Naval Research, the National Science Foundation and the Army Research Office is gratefully acknowledged. We appreciate the help of Shaoping Xiao with the molecular mechanics simulations.

\section{Appendix A. 2-body/3-body potentials for graphene sheets}

\section{A.1. Crystal structure}

Carbon nanotubes can be viewed as graphene sheets rolled into a tube. The undeformed bond network can be characterized by the three bond vectors of length $A_{0}$ forming an angle of $2 \pi / 3$ between them: $\mathbf{A}_{0}^{i}, i=1,2,3$. The bond vectors affected by the inner displacements are (see Fig. 6):

$$
\mathbf{A}^{i}=\mathbf{A}_{0}^{i}+\boldsymbol{\eta} .
$$

The relative orientation of the original bond vectors $\mathbf{A}_{0}^{i}$ determines the chirality of the nanotube. For instance, when one of these vectors is parallel to the nanotube axis, it is called a zig-zag nanotube (Saito et al., 1992). On the other hand, when one is perpendicular to the axis, we have an arm-chair nanotube.

\section{A.2. Strain energy density}

To develop an expression for the strain energy density of the nanotube based on the inter-atomic potentials, a homogenization process at the level of the RVE is carried out. The strain energy density considered is per unit reference area. The homogenization process is performed in the reference state, that corresponds to the graphene sheet in equilibrium. We will consider the instance in which the atomic system is governed by potentials of the type described in Eq. (43).

The hexagonal cell of the crystal is a RVE for the closest neighbor interaction potentials considered. The area of the hexagonal cell in the undeformed graphene sheet is $S_{0}=(3 \sqrt{3} / 2) A_{0}^{2}$. In each cell there is one bond of each type and two angles of each type. Indeed, it is easy to show from the expressions in the paper that, on the one hand, the length of the deformed bonds cortrresponding to $\mathbf{A}^{i}$ and to $-\mathbf{A}^{i}$ is the same, and on the other hand the angle that the deformed bonds corresponding to $\mathbf{A}^{i}$ and $\mathbf{A}^{j}$ form is equal to the angle corresponding to $-\mathbf{A}^{i}$ and $-\mathbf{A}^{j}$. 
Therefore, recalling Eq. (46), the strain energy is

$$
W(\mathbf{C}, \mathscr{K}, \boldsymbol{\eta})=\frac{1}{S_{0}}\left[\sum_{l=1}^{3} \mathscr{V}_{s}\left(\left\|\mathbf{a}^{l}\right\|\right)+2 \sum_{k=1}^{3} \mathscr{V}_{\theta}\left(\theta^{k},\left\|\mathbf{a}^{i}\right\|,\left\|\mathbf{a}^{j}\right\|\right)\right],
$$

where $\{i, j, k\}$ is an even permutation of $\{1,2,3\}$. Its dependence on the continuum strain measures $\mathbf{C}$ and $\mathscr{K}$ and the inner displacements $\boldsymbol{\eta}$ has been stressed, and can be traced through Eqs. (A.1) and (40). Now it suffices to follow the procedure described in Section 6.1.3 to eliminate the inner displacements (now these are a continuum internal variable) and obtain $\hat{W}(\mathbf{C}, \mathscr{K})$.

\section{A.3. Summary of the evaluation of the strain energy density}

Recall that the undeformed body is planar and described with Euclidean coordinates $\left\{X_{1}, X_{2}\right\}$. Because of this, cova- and contra- components of tensors are indistinguishable, and so are the tensors $\mathbf{C}$ and $\mathbf{C}^{b}$. Their matrix representation in the orthonormal basis $\mathscr{B}_{\mathbb{R}^{2}}$ associated to $\left\{X_{1}, X_{2}\right\},[\mathbf{C}]=\left[\mathbf{C}^{b}\right]$ is symmetric, as well as $[\mathscr{K}]$. To keep the notation simple, we do not distinguish between the vectors and tensors themselves and their representation in this Euclidean coordinate system of $\Omega_{0}$. Also, the notation $\{\cdot\}^{A}$ means extracting the $A$ th component of a vector in this Euclidean coordinate system. In our total Lagrangian formulation, all quantities are expressed in the undeformed body.

Assume we are given the strain measures $\mathbf{C}$ and $\mathscr{K}$ at a point $\mathbf{X} \in \Omega_{0}$, whose components are denoted as

$$
\mathbf{C}=\left[\begin{array}{ll}
E & F \\
F & G
\end{array}\right] \text { and } \mathscr{K}=\left[\begin{array}{cc}
L & M \\
M & N
\end{array}\right] .
$$

Suppose we are also given the inner displacements $\boldsymbol{\eta}$ at the point $\mathbf{X}$ of the undeformed solid. Finally, the orientation of the undeformed lattice is given with respect to the coordinate system, and therefore the three undeformed bond vectors $\mathbf{A}_{0}^{i}$ are known. The bond vectors perturbed with the inner displacements are denoted $\mathbf{A}^{i}=\mathbf{A}_{0}^{i}+\boldsymbol{\eta}$, and $A^{i}$ denotes the length of each of these vectors. Three unit vectors are then defined,

$$
\mathbf{E}^{i}=\frac{1}{A^{i}} \mathbf{A}^{i}
$$

The mean and the Gaussian curvatures of the deformed membrane can be computed as

$$
H=\frac{1}{2} \operatorname{trace}\left(\mathbf{C}^{-1} \mathscr{K}\right)=\frac{1}{2} \mathbf{C}^{-1}: \mathscr{K} \quad \text { and } \quad K=\frac{\operatorname{det} \mathscr{K}}{\operatorname{det} \mathbf{C}}
$$

and the principal curvatures as

$$
k_{I, I I}=H \pm \sqrt{H^{2}-K} .
$$

The principal directions expressed in $\Omega_{0}$ are then obtained from Eq. (24):

$$
\mathbf{V}_{I, I I}=\left\{\begin{array}{c}
-M+k_{I, I I} F \\
L-k_{I, I I} E
\end{array}\right\}+\alpha_{I, I I}\left\{\begin{array}{c}
32 \\
-N+k_{I, I I} G \\
M-k_{I, I I} F
\end{array}\right\}
$$


where the scalar parameters $\alpha_{I, I I}$ are set, for instance, to zero or one, to ensure $\mathbf{V}_{I, I I}$ are not (close to) zero. If the deformed surface is planar, the above expression does not work, and any two $\mathbf{C}$-orthogonal vectors of $\Omega_{0}$ can be chosen.

In Section 5.1, we introduced the auxiliary quantities $w_{I, I I}$, the components of the deformed tangent vectors in a local coordinate system of the tangent of the deformed body. Now, each of the three bond vectors has a corresponding deformed tangent vector $\mathbf{w}^{i}=\mathbf{F} \mathbf{A}^{i}$, and their components are,

$$
w_{I, I I}^{i}=A^{i} \frac{\mathbf{V}_{I, I I} \mathbf{C} \mathbf{E}^{i}}{\sqrt{\mathbf{V}_{I, I I} \mathbf{C} \mathbf{V}_{I, I I}}}=A^{i} \frac{\left\langle\mathbf{V}_{I, I I} \mid \mathbf{E}^{i}\right\rangle_{\mathbf{C}}}{\left\|\mathbf{V}_{I, I I}\right\|_{\mathbf{C}}} .
$$

Recalling Eq. (40), where the deformed lattice vector a was expressed in an orthonormal basis, we can compute the scalar products between the three deformed bond vectors,

$$
\begin{aligned}
\left\langle\mathbf{a}^{i} \mid \mathbf{a}^{j}\right\rangle= & w_{I}^{i} \mathscr{Q}\left(k_{I} w_{I}^{i}\right) w_{I}^{j} \mathscr{Q}\left(k_{I} w_{I}^{j}\right)+w_{I I}^{i} \mathscr{Q}\left(k_{I I} w_{I I}^{i}\right) w_{I I}^{j} \mathscr{Q}\left(k_{I I} w_{I I}^{j}\right) \\
& +\left[\frac{k_{I}\left(w_{I}^{i}\right)^{2}}{2} \mathscr{Q}^{2}\left(k_{I} w_{I}^{i} / 2\right)+\frac{k_{I I}\left(w_{I I}^{i}\right)^{2}}{2} \mathscr{Q}^{2}\left(k_{I I} w_{I I}^{i} / 2\right)\right] \\
& \times\left[\frac{k_{I}\left(w_{I}^{j}\right)^{2}}{2} \mathscr{Q}^{2}\left(k_{I} w_{I}^{j} / 2\right)+\frac{k_{I I}\left(w_{I I}^{j}\right)^{2}}{2} \mathscr{Q}^{2}\left(k_{I I} w_{I I}^{j} / 2\right)\right] .
\end{aligned}
$$

Finally, the lengths and angles of the deformed bond vectors can be computed from these scalar products:

$$
\left\|\mathbf{a}^{l}\right\|=\sqrt{\left\langle\mathbf{a}^{l} \mid \mathbf{a}^{l}\right\rangle} \quad \text { and } \quad \theta^{k}=\arccos \frac{\left\langle\mathbf{a}^{i} \mid \mathbf{a}^{j}\right\rangle}{\left\|\mathbf{a}^{i}\right\|\left\|\mathbf{a}^{j}\right\|},
$$

where $\{i, j, k\}$ is an even permutation of $\{1,2,3\}$. Then, these quantities can be plugged into Eq. (A.2) to obtain the strain energy density of the elastic solid.

\section{A.4. Stress tensors}

Recalling the expression of the strain energy density (A.2) and evaluating everything at the relaxed inner displacements $\hat{\boldsymbol{\eta}}$, the stress measures can be written as

$$
\begin{aligned}
\mathbf{n}= & 2 \frac{\partial \hat{W}}{\partial \mathbf{C}}=2 \frac{\partial W}{\partial \mathbf{C}} \\
= & \frac{2}{S_{0}}\left[\sum_{l=1}^{3} \mathscr{V}_{s}^{\prime} \frac{\partial\left\|\mathbf{a}_{l}\right\|}{\partial \mathbf{C}}+2 \sum_{k=1}^{3}\left(\frac{\partial \mathscr{V}_{\theta}}{\partial \theta} \frac{\partial \theta_{k}}{\partial \mathbf{C}}\right.\right. \\
& \left.\left.+\frac{\partial \mathscr{V}_{\theta}}{\partial r^{1}} \frac{\partial\left\|\mathbf{a}_{i}\right\|}{\partial \mathbf{C}}+\frac{\partial \mathscr{V}_{\theta}}{\partial r^{2}} \frac{\partial\left\|\mathbf{a}_{j}\right\|}{\partial \mathbf{C}}\right)\right]^{33},
\end{aligned}
$$


and

$$
\begin{aligned}
\mathbf{m}= & \frac{\partial \hat{W}}{\partial \mathscr{K}}=\frac{\partial W}{\partial \mathscr{K}} \\
= & \frac{1}{S_{0}}\left[\sum_{l=1}^{3} \mathscr{V}_{s}^{\prime} \frac{\partial\left\|\mathbf{a}_{l}\right\|}{\partial \mathscr{K}}+2 \sum_{k=1}^{3}\left(\frac{\partial \mathscr{V}_{\theta}}{\partial \theta} \frac{\partial \theta_{k}}{\partial \mathscr{K}}+\frac{\partial \mathscr{V}_{\theta}}{\partial r^{1}} \frac{\partial\left\|\mathbf{a}_{i}\right\|}{\partial \mathscr{K}}\right.\right. \\
& \left.\left.+\frac{\partial \mathscr{V}_{\theta}}{\partial r^{2}} \frac{\partial\left\|\mathbf{a}_{j}\right\|}{\partial \mathscr{K}}\right)\right] .
\end{aligned}
$$

In the following, several expressions needed in the computation of the stress measures are provided. The derivatives of the mean and the Gaussian curvatures with respect to the strain measures can be written as

$$
\begin{aligned}
& \frac{\partial H}{\partial \mathbf{C}}=-\frac{1}{2} \mathbf{C}^{-1} \mathscr{K} \mathbf{C}^{-1}, \quad \frac{\partial K}{\partial \mathbf{C}}=-K \mathbf{C}^{-1}, \\
& \frac{\partial H}{\partial \mathscr{K}}=\frac{1}{2} \mathbf{C}^{-1}, \quad \frac{\partial K}{\partial \mathscr{K}}=K \mathscr{K}^{-1} .
\end{aligned}
$$

The derivatives of the principal curvatures with respect to the strain measures are then:

$$
\frac{\partial k_{I, I I}}{\partial \mathbf{Z}}=\frac{\partial H}{\partial \mathbf{Z}} \pm \frac{1}{2 \sqrt{H^{2}-K}}\left(2 H \frac{\partial H}{\partial \mathbf{Z}}-\frac{\partial K}{\partial \mathbf{Z}}\right),
$$

where $\mathbf{Z}$ denotes either $\mathbf{C}$ or $\mathscr{K}$.

When it comes to the derivatives of the principal directions, let us introduce the following matrices:

$$
\mathbf{M}_{1}=\left[\begin{array}{cc}
0 & 1 / 2 \\
1 / 2 & 0
\end{array}\right], \quad \mathbf{M}_{2}=\left[\begin{array}{ll}
1 & 0 \\
0 & 0
\end{array}\right] \quad \text { and } \quad \mathbf{M}_{3}=\left[\begin{array}{ll}
0 & 0 \\
0 & 1
\end{array}\right] .
$$

The derivative with respect to the Green deformation tensor is then

$$
\frac{\partial \mathbf{V}_{I, I I}}{\partial \mathbf{C}}=\left\{\begin{array}{c}
F \frac{\partial k_{I, I I}}{\partial \mathbf{C}}+k_{I, I I} \mathbf{M}_{1} \\
-E \frac{\partial k_{I, I I}}{\partial \mathbf{C}}-k_{I, I I} \mathbf{M}_{2}
\end{array}\right\}+\alpha_{I, I I}\left\{\begin{array}{c}
G \frac{\partial k_{I, I I}}{\partial \mathbf{C}}+k_{I, I I} \mathbf{M}_{3} \\
-F \frac{\partial k_{I, I I}}{\partial \mathbf{C}}-k_{I, I I} \mathbf{M}_{1}
\end{array}\right\}
$$

and that with respect to the curvature is

$$
\frac{\partial \mathbf{V}_{I, I I}}{\partial \mathscr{K}}=\left\{\begin{array}{c}
F \frac{\partial k_{I, I I}}{\partial \mathscr{K}}-\mathbf{M}_{1} \\
-E \frac{\partial k_{I, I I}}{\partial \mathscr{K}}+\mathbf{M}_{2}
\end{array}\right\}+\alpha_{I, I I}^{34}\left\{\begin{array}{c}
G \frac{\partial k_{I, I I}}{\partial \mathscr{K}}-\mathbf{M}_{3} \\
-F \frac{\partial k_{I, I I}}{\partial \mathscr{K}}+\mathbf{M}_{1}
\end{array}\right\} .
$$


Noting the following expressions:

$$
\begin{aligned}
& \frac{\partial\left\|\mathbf{V}_{I, I I}\right\|_{\mathbf{C}}}{\partial \mathbf{C}}=\frac{1}{\left\|\mathbf{V}_{I, I I}\right\|_{\mathbf{C}}}\left[\frac{\partial\left\{\mathbf{V}_{I, I I}\right\}^{A}}{\partial \mathbf{C}}\left\{\mathbf{C} \mathbf{V}_{I, I I}\right\}^{A}+\frac{1}{2} \mathbf{V}_{I, I I} \otimes \mathbf{V}_{I, I I}\right] \\
& \frac{\partial\left\langle\mathbf{V}_{I, I I} \mid \mathbf{E}^{i}\right\rangle_{\mathbf{C}}}{\partial \mathbf{C}}=\frac{\partial\left\{\mathbf{V}_{I, I I}\right\}^{A}}{\partial \mathbf{C}}\left\{\mathbf{C} \mathbf{E}^{i}\right\}^{A}+\frac{1}{2}\left(\mathbf{V}_{I, I I} \otimes \mathbf{E}^{i}+\mathbf{E}^{i} \otimes \mathbf{V}_{I, I I}\right), \\
& \frac{\partial\left\|\mathbf{V}_{I, I I}\right\|_{\mathbf{C}}}{\partial \mathscr{K}}=\frac{1}{\left\|\mathbf{V}_{I, I I}\right\|_{\mathbf{C}}} \frac{\partial\left\{\mathbf{V}_{I, I I}\right\}^{A}}{\partial \mathscr{K}}\left\{\mathbf{C} \mathbf{V}_{I, I I}\right\}^{A}
\end{aligned}
$$

and

$$
\frac{\partial\left\langle\mathbf{V}_{I, I I} \mid \mathbf{E}^{i}\right\rangle_{\mathbf{C}}}{\partial \mathscr{K}}=\frac{\partial\left\{\mathbf{V}_{I, I I}\right\}^{A}}{\partial \mathscr{K}}\left\{\mathbf{C E}^{i}\right\}^{A}
$$

where the summation convention applies to the index $A=1,2$, the derivatives of $w_{I, I I}^{i}$ with respect to the Green deformation and curvature tensors can be computed as

$$
\frac{\partial w_{I, I I}^{i}}{\partial \mathbf{Z}}=A^{i} \frac{1}{\left\|\mathbf{V}_{I, I I}\right\|_{\mathbf{C}}^{2}}\left(\left\|\mathbf{V}_{I, I I}\right\|_{\mathbf{C}} \frac{\partial\left\langle\mathbf{V}_{I, I I} \mid \mathbf{E}^{i}\right\rangle_{\mathbf{C}}}{\partial \mathbf{Z}}-\left\langle\mathbf{V}_{I, I I} \mid \mathbf{E}^{i}\right\rangle_{\mathbf{C}} \frac{\partial\left\|\mathbf{V}_{I, I I}\right\|_{\mathbf{C}}}{\partial \mathbf{Z}}\right),
$$

where $\mathbf{Z}$ denotes either $\mathbf{C}$ or $\mathscr{K}$.

The above expressions can be used to compute the derivatives of the scalar products $\left\langle\mathbf{a}^{i} \mid \mathbf{a}^{j}\right\rangle$ with respect to the strain measures, and therefore readily obtain the corresponding stress tensors. There is however one more detail to be addressed. Remark 4 stressed the need of a careful implementation of the function $2(x)=\sin x / x$ as $x$ is close to zero. Similarly, the derivative of this function $\mathscr{2}^{\prime}(x)=(\cos x-\sin x / x) / x$, which appears in the final formula for the stresses, needs to be implemented with care to avoid numerical inaccuracy. Also in this case, a Taylor series expansion is used when $x$ is smaller than a certain threshold.

\section{A.5. Inner stress}

The derivative of the strain energy density $W(\mathbf{C}, \mathscr{K}, \boldsymbol{\eta})$ with respect to the inner displacements is needed for the local relaxation that yields $\hat{W}(\mathbf{C}, \mathscr{K})$, and can be called "inner stress":

$$
\begin{aligned}
\mathbf{h}=\frac{\partial W}{\partial \boldsymbol{\eta}}= & \frac{1}{S_{0}}\left[\sum_{l=1}^{3} \mathscr{V}_{s}^{\prime} \frac{\partial\left\|\mathbf{a}_{l}\right\|}{\partial \boldsymbol{\eta}}+2 \sum_{k=1}^{3}\left(\frac{\partial \mathscr{V}_{\theta}}{\partial \theta} \frac{\partial \theta_{k}}{\partial \boldsymbol{\eta}}\right.\right. \\
& \left.\left.+\frac{\partial \mathscr{V}_{\theta}}{\partial r^{1}} \frac{\partial\left\|\mathbf{a}_{i}\right\|}{\partial \boldsymbol{\eta}}+\frac{\partial \mathscr{V}_{\theta}}{\partial r^{2}} \frac{\partial\left\|\mathbf{a}_{j}\right\|}{\partial \boldsymbol{\eta}}\right)\right] .
\end{aligned}
$$

In this section, several useful expressions for its computation are provided.

It can be easily seen that

$$
\frac{\partial \mathbf{A}^{i}}{\partial \boldsymbol{\eta}}=\mathbf{I d}_{2 \times 2}
$$


Recall that the quantities $w_{I, I I}^{i}$ are the components of the vector $\mathbf{w}^{i}=\mathbf{F} \mathbf{A}^{i}$ in the basis $\mathscr{B}_{T_{\mathbf{x}} \Omega}=\left\{\mathbf{i}_{I}, \mathbf{i}_{I I}\right\}$ introduced in Section 5.1. Therefore,

$$
\begin{aligned}
w_{I, I I}^{i} & =\left\langle\mathbf{w}^{i} \mid \mathbf{i}_{I, I I}\right\rangle=\left\langle\mathbf{w}^{i} \mid \frac{1}{\left\|\mathbf{v}_{I, I I}\right\|} \mathbf{v}_{I, I I}\right\rangle=\frac{1}{\left\|\mathbf{V}_{I, I I}\right\|_{\mathbf{C}}}\left\langle\mathbf{A}^{i} \mid \mathbf{V}_{I, I I}\right\rangle_{\mathbf{C}} \\
& =\frac{1}{\left\|\mathbf{V}_{I, I I}\right\|_{\mathbf{C}}} \mathbf{A}^{i} \mathbf{C} \mathbf{V}_{I, I I} .
\end{aligned}
$$

Consequently, one can show that, irrespective of the bond index $i$, we have

$$
\frac{\partial w_{I, I I}^{i}}{\partial \boldsymbol{\eta}}=\frac{1}{\left\|\mathbf{V}_{I, I I}\right\|_{\mathbf{C}}} \mathbf{C} \mathbf{V}_{I, I I} .
$$

This can be used to compute the derivative the deformed bond vector a expressed in the orthonormal basis described in Section 5.4:

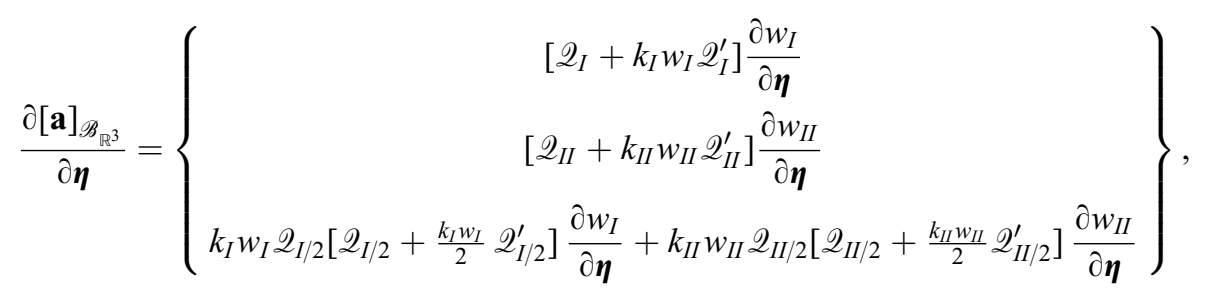

where the following notations have been used:

$$
\begin{aligned}
& \mathscr{Q}_{I, I I}=\mathscr{2}\left(k_{I, I I} w_{I, I I}\right), \quad \mathscr{Q}_{I, I I / 2}=\mathscr{2}\left(k_{I, I I} w_{I, I I} / 2\right), \\
& \mathscr{Q}_{I, I I}^{\prime}=\mathscr{Q}^{\prime}\left(k_{I, I I} w_{I, I I}\right), \quad \mathscr{Q}_{I, I I / 2}^{\prime}=\mathscr{Q}^{\prime}\left(k_{I, I I} w_{I, I I} / 2\right) .
\end{aligned}
$$

With these expressions, the calculation of the "inner stress" $\mathbf{h}$ becomes straightforward.

\section{References}

Arroyo, M., Belytschko, T., 2001. A finite deformation membrane based on inter-atomic potentials for the transverse mechanics of nanotubes. Mech. Mater., in press.

Belytschko, T., Xiao, S., Schatz, G., Ruoff, R., 2001. Atomistic simulation of nanotubes fracture. Phys. Rev. $\mathrm{B}$, in press.

Bernholc, J., Brabec, C., Nardelli, M., Maiti, A., Roland, C., Yakobson, B., 1998. Theory of growth and mechanical properties of nanotubes. Appl. Phys. A 67, 39-46.

Brenner, D., 1990. Empirical potential for hydrocarbons for use in simulating chemical vapor deposition of diamond films. Phys. Rev. B 42 (15), 9458-9471.

Chopra, N., Benedict, L., Crespi, V., Cohen, M., Louie, S., Zettl, A., 1995. Fully collapsed carbon nanotubes. Nature 377, 135-138.

Cirak, F., Ortiz, M., 2001. Fully $C^{1}$-conforming subdivision elements for finite deformation thin-shell analysis. Int. J. Numer. Meth. Eng. 51 (7), 813-833.

Cirak, F., Ortiz, M., Schröder, P., 2000. Subdivision surfaces: a new paradigm for thin-shell finite-element analysis. Int. J. Numer. Meth. Eng. 47, 2039-20726

Cousins, C., 1978. Inner elasticity. J. Phys. C 11, 4867-4879.

Dacorogna, B., 1989. Direct Methods in the Calculus of Variations. Vol. 78, Applied Mathematical Sciences. Springer-Verlag, p. 276. 
do Carmo, M.P., 1976. Differential Geometry of Curves and Surfaces. Prentice-Hall, Englewood Cliffs, NJ.

Ericksen, J.L., 1984. The Cauchy and Born Hypotheses for Crystals. In: Gurtin, M.E. (Ed.), Phase transformations and material instabilities in solids. Academic Press, New York, pp. 61-77.

Falvo, M.R., Clary, G.J., Taylor, R.M., Chi, V., Brooks, F.P., Washburn, S., Superfine, R., 1997. Bending and buckling of carbon nanotubes under large strain. Nature 389, 582-584.

Friesecke, G., James, R., 2000. A scheme for the passage from atomic to continuum theory for thin films, nanotubes and nanorods. J. Mech. Phys. Solids 48, 1519-1540.

Gao, G., Çağin, T., Goddard III, W., 1998. Energetics, structure, mechanical and vibrational properties of single-walled carbon nanotubes. Nanotechnology 9, 184-191.

Gilbert, J., Nocedal, J., 1992. Global convergence properties of conjugate gradient methods for optimization SIAM J. Optim. 2 (1).

Lu, J.P., 1997. Elastic properties of carbon nanotubes and nanoropes. Phys. Rev. Lett. 79 (7), 1297-1300.

Maiti, A., 2000. Mechanical deformation in carbon nanotubes- bent tubes vs tubes pushed by atomically sharp tips. Chem. Phys. Lett. 331, 21-25.

Malvern, L.E., 1969. Introduction to the Mechanics of a Continuous Medium. Prentice-Hall, Englewood Cliffs, NJ.

Marsden, J.E., Hughes, T.J., 1983. Mathematical Foundations of Elasticity. Prentice-Hall, Englewood Cliffs, NJ.

Martin, J., 1975. Many-body forces in metals and the brugger elastic constants. J. Phys. C 8, 2837-2857.

Morgan, F., 1993. Riemannian Geometry, a Beginner's Guide. Jones and Bartlett Publishers, Boston, MA.

Ru, C., 2001. Axially compressed buckling of a doublewalled carbon nanotube embedded in an elastic medium. J. Mech. Phys. Solids 49, 1265-1279.

Saito, R., Fujita, M., Dresselhaus, G., Dresselhaus, M., 1992. Electronic structure of chiral graphene tubules. Appl. Phys. Lett. 60 (18), 2204-2206.

Shenoy, V., Miller, R., Tadmor, E., Rodney, D., Phillips, R., Ortiz, M., 1999. An adaptive finite element approach to atomic-scale mechanics - the quasicontinuum method. J. Mech. Phys. Solids 47, 611-642.

Simo, J., Fox, D., 1989. On a stress resultant geometrically exact shell model. Part i: Formulation and optimal parametrization. Comput. Meth. Appl. Mech. Eng. 72, 267-304.

Simo, J., Hughes, T., 1998. Computational Inelasticity. Springer, Berlin.

Sohlberg, K., Sumpter, B., Tuzun, R., Noid, D., 1998. Continuum methods of mechanics as a simplified approach to structural engineering of nanostructures. Nanotechnology 9, 30-36.

Tadmor, E., Ortiz, M., Phillips, R., 1996. Quasicontinuum analysis of defects in solids. Philos. Mag. A 73 (6), 1529-1563.

Tadmor, E., Smith, G., Bernstein, N., Kaxiras, E., 1999. Mixed finite element and atomistic formulation for complex crystals. Phys. Rev. B 59 (1), 235-245.

Yakobson, B., Brabec, C., Bernholc, J., 1996. Nanomechanics of carbon tubes: instabilities beyond the linear response. Phys. Rev. Lett. 76 (14), 2511-2514.

Yu, M., Lourie, O., Dyer, M., Moloni, K., Kelly, T., Ruoff, R., 2000. Strength and breaking mechanism of multiwalled carbon nanotubes under tensile load. Science 287, 637-640.

Yu, M., Dyer, M., Ruoff, R., 2001a. Structure and mechanical flexibility of carbon nanotube ribbons: an atomic-force microscopy study. J. Appl. Phys. 89 (8), 4554-4557.

Yu, M., Kowalewski, T., Ruoff, R., 2001b. Structural analysis of collapsed, and twisted and collapsed, multiwalled carbon nanotubes by atomic force microscopy. Phys. Rev. Lett. 86 (1), 87-90.

Zanzotto, G., 1996. The Cauchy-Born hypothesis, nonlinear elasticity and mechanical twinning in crystals. Acta Crystallogr. A 52, 839-849.

Zhong-can, O.-Y., Su, Z.-B., Wang, C.-L., 1997. Coil formation in multishell carbon nanotubes: competition between curvature elasticity and interlayer adhesion. Phys. Rev. Lett. 78 (21), 4055-4058.

Zhou, G., Duan, W., Gu, B., 2001. First-principles study on morphology and mechanical properties of single-walled carbon nanotube. Chem. Phys. Lett. 333, 344-349. 